\title{
The HOSTS Survey-Exozodiacal Dust Measurements for 30 Stars
}

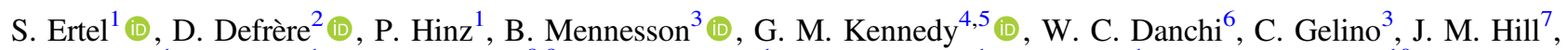

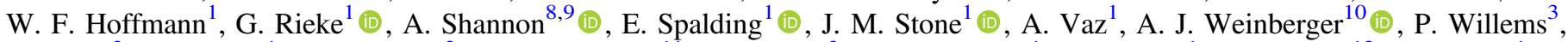
O. Absil ${ }^{2}$ (1) P. Arbo ${ }^{1}$, V. P. Bailey ${ }^{3}$ (10, C. Beichman ${ }^{11}$, G. Bryden ${ }^{3}$, E. C. Downey ${ }^{1}$, O. Durney ${ }^{1}$, S. Esposito ${ }^{12}$, A. Gaspar ${ }^{1}$ (1), P. Grenz ${ }^{1}$, C. A. Haniff ${ }^{13}$, J. M. Leisenring ${ }^{1}(\mathbb{1})$, L. Marion ${ }^{2}$, T. J. McMahon ${ }^{1}$, R. Millan-Gabet ${ }^{11}$ (1), M. Montoya ${ }^{1}$, K. M. Morzinski ${ }^{1}$ (10, E. Pinna ${ }^{12}$ (1) , J. Power ${ }^{7}$, A. Puglisi ${ }^{12}$, A. Roberge ${ }^{6}$ (i), E. Serabyn ${ }^{3}$, A. J. Skemer ${ }^{14}$ (10, K. Stapelfeldt ${ }^{3}$, K. Y. L. Su ${ }^{1}$ (1), V. Vaitheeswaran ${ }^{1}$, and M. C. Wyatt ${ }^{5}$ (1)

\footnotetext{
${ }^{1}$ Steward Observatory, Department of Astronomy, University of Arizona, 993 N. Cherry Ave, Tucson, AZ 85721, USA; sertel@email.arizona.edu

${ }_{2}^{2}$ Space Sciences, Technologies \& Astrophysics Research (STAR) Institute, University of Liège, Liège, Belgium

${ }^{3}$ Jet Propulsion Laboratory, California Institute of Technology, 4800 Oak Grove Dr., Pasadena, CA 91109, USA

${ }^{4}$ Department of Physics, University of Warwick, Gibbet Hill Road, Coventry CV4 7AL, UK

${ }^{5}$ Institute of Astronomy, University of Cambridge, Madingley Road, Cambridge CB3 0HA, UK

${ }^{6}$ NASA Goddard Space Flight Center, Exoplanets \& Stellar Astrophysics Laboratory, Code 667, Greenbelt, MD 20771, USA

${ }^{7}$ Large Binocular Telescope Observatory, University of Arizona, 933 N. Cherry Avenue, Tucson, AZ 85721, USA

${ }_{9}^{8}$ Department of Astronomy and Astrophysics, The Pennsylvania State University, State College, PA 16801, USA

${ }^{9}$ Center for Exoplanets and Habitable Worlds, The Pennsylvania State University, State College, PA 16802, USA

${ }^{10}$ Department of Terrestrial Magnetism, Carnegie Institution of Washington, 5241 Broad Branch Road NW, Washington, DC 20015, USA

${ }^{11}$ NASA Exoplanet Science Institute, MS 100-22, California Institute of Technology, Pasadena, CA 91125, USA

${ }^{12}$ INAF-Osservatorio Astrofisico di Arcetri, Largo E. Fermi 5, I-50125 Firenze, Italy

${ }^{13}$ Cavendish Laboratory, University of Cambridge, JJ Thomson Avenue, Cambridge CB3 0HE, UK

${ }^{14}$ Astronomy Department, University of California Santa Cruz, 1156 High Street, Santa Cruz, CA 95064, USA

Received 2017 December 10; revised 2018 March 9; accepted 2018 March 13; published 2018 April 17
}

\begin{abstract}
The Hunt for Observable Signatures of Terrestrial Systems survey searches for dust near the habitable zones (HZs) around nearby, bright main-sequence stars. We use nulling interferometry in the $N$ band to suppress the bright stellar light and to probe for low levels of $\mathrm{HZ}$ dust around the 30 stars observed so far. Our overall detection rate is $18 \%$, including four new detections, among which are the first three around Sun-like stars and the first two around stars without any previously known circumstellar dust. The inferred occurrence rates are comparable for early-type and Sun-like stars, but decrease from $60_{-21}^{+16} \%$ for stars with previously detected cold dust to $8_{-3}^{+10} \%$ for stars without such excess, confirming earlier results at higher sensitivity. For completed observations on individual stars, our sensitivity is five to ten times better than previous results. Assuming a lognormal excess luminosity function, we put upper limits on the median HZ dust level of 13 zodis (95\% confidence) for a sample of stars without cold dust and of 26 zodis when focusing on Sun-like stars without cold dust. However, our data suggest that a more complex luminosity function may be more appropriate. For stars without detectable Large Binocular Telescope Interferometer (LBTI) excess, our upper limits are almost reduced by a factor of two, demonstrating the strength of LBTI target vetting for future exo-Earth imaging missions. Our statistics are limited so far, and extending the survey is critical to informing the design of future exo-Earth imaging surveys.
\end{abstract}

Key words: circumstellar matter - infrared: stars - planetary systems - techniques: interferometric - zodiacal dust

\section{Introduction}

Exozodiacal dust-exozodi for short-is warm and hot dust (temperatures between a few $100 \mathrm{~K}$ and $\sim 2000 \mathrm{~K}$ ) around main-sequence stars. In analogy to zodiacal dust in the solar system, the term refers to dust near the habitable zone (HZ) of the host star and closer in. It is produced through asteroid collisions (Dermott et al. 2002) and comet evaporation (Nesvorný et al. 2010) and is redistributed under the influence of additional collisions, stellar radiation, wind, and magnetic fields, as well as through interaction with any nearby planets (e.g., Wyatt 2005; Stark \& Kuchner 2008; Brogi et al. 2009; Reidemeister et al. 2011; Ertel et al. 2012; van Lieshout et al. 2014; Kennedy \& Piette 2015; Rieke et al. 2016). Thus, studying the dust gives insight into the architecture and dynamics of planetary systems in their inner regions, including the HZ.

At the same time, the potential presence of large amounts of HZ dust around nearby stars is a dominant source of uncertainty for planning future exo-Earth imaging missions
(Roberge et al. 2012). The typical amount of dust present determines the size of the primary mirror(s) needed to detect exo-Earths with a coronagraph or starshade in the visible (e.g., Stark et al. 2015, 2016) or a mid-infrared nulling interferometer (Defrère et al. 2010). Characterizing the occurrence rate of the dust and its potential correlation with more accessible properties of the systems such as stellar spectral type, age, or the presence of massive Kuiper Belt analogs is vital for the design and target selection of such missions, and thus for their success.

Because of its high temperature compared to colder, Kuiper Belt-like debris disks, exozodiacal dust emits predominantly in the near- and mid-infrared (nIR and mIR), where aside from a few exceptional cases it is outshone by the host star. The ability of photometry and low-resolution spectroscopy to disentangle disk and stellar emission is limited by uncertainties from calibration and the prediction of the stellar photospheric flux, such that their typical sensitivity limits are of the order of a few percent of the stellar flux (Beichman et al. 2006). Detecting scattered light from the dust requires extreme contrast very close to the star. Targeted coronagraphic observations with the 
Wide-field Infrared Survey Telescope (Krist et al. 2016) may be able to image a few systems, but potential targets need to be identified first. Given the small angular scales involved (1 au at $10 \mathrm{pc}$ corresponds to 0 .' 1 ), only infrared interferometry currently provides the angular resolution and contrast needed to spatially disentangle the dust emission from the stellar emission, enabling the detection of faint excesses. Optical longbaseline interferometry in the nIR has been very successful in detecting and characterizing hot dust very close to nearby main-sequence stars (Absil et al. 2006, 2013; Defrère et al. 2012; Ertel et al. 2014, 2016; Nuñez et al. 2017; Marion et al. 2018), but its connection to HZ dust is still unclear. On the other hand, the emission of warm $\mathrm{HZ}$ dust with a temperature of $\sim 300 \mathrm{~K}$ peaks in the mIR, where nulling interferometry is currently the most sensitive method to detect it. With this technique, the light from the central star is brought to destructive interference, producing a central dark fringe, while spatially resolved emission is transmitted.

This method was used for a first dedicated exozodi survey by the Keck Interferometer Nuller (KIN; Millan-Gabet et al. 2011; Serabyn et al. 2012; Mennesson et al. 2013). While critical constraints on the occurrence of bright exozodiacal dust were derived, the detection of dust levels comparable to the solar system was out of reach, and the results could not sufficiently inform the design of future exo-Earth imaging missions. To go beyond these results, we have developed the Large Binocular Telescope Interferometer (LBTI; Hinz et al. 2016) and its mIR nulling mode. We are carrying out the HOSTS (Hunt for Observable Signatures of Terrestrial Systems; Danchi et al. 2014) survey. In this paper, we present the statistical results from the 30 individual stars observed so far. We provide the strongest constraints on the incidence rate and typical brightness of $\mathrm{HZ}$ dust. They are particularly timely because they provide valuable input for NASA's 2020 decadal survey, during which mission concepts potentially capable of exo-Earth imaging will be evaluated (HabEx, Mennesson et al. 2016b; LUVOIR, Crooke et al. 2016).

We discuss the sample of stars included in this paper in Section 2. Our instrument and observing strategy are described in Section 3. The data reduction and detection methods are described in Section 4. Our results are presented in Section 5 and discussed in Section 6. Our conclusions are presented in Section 7.

\section{Sample}

The full target list of the HOSTS survey has been discussed in detail by Weinberger et al. (2015). In short, it consists of nearby, bright main-sequence stars ( $>1 \mathrm{Jy}$ in $N$ band) without known close $(<1$ ".5) binary companions. The sample is separated into early-type stars (spectral types A to F5), for which our observations are most sensitive, and Sun-like stars (spectral types F6 to K8), which are preferred targets for future exo-Earth imaging missions. The combination of the two groups allows us to probe the incidence rate of exozodiacal dust across a large range of stellar masses, thereby providing access to the physical processes at play for its production and evolution. In order to provide the strongest intermediate results at any time, we maintain a balanced sample between early-type and Sun-like stars during the observations. Table 1 lists the basic, relevant properties of the targets observed so far. For a fraction of the stars, the observations have been completed (three or four calibrated science pointings obtained). For the other stars, more data will be obtained, so the final null measurements are expected to differ within the uncertainties from the values presented here, and the final uncertainties will be smaller than the ones in the present work.

Calibrators were selected following Mennesson et al. (2014) using the catalogs of Bordé et al. (2002) and Mérand et al. (2005), supplemented by stars from the JSDC catalog and the SearchCal tool (both Chelli et al. 2016) where necessary. A minimum of three (in most cases four) different stars were used to calibrate the observations of a single science target (Section 3.2) to minimize systematics due to imperfect knowledge of the calibrators (uncertain diameters, potential multiplicity, or faint circumstellar emission).

\section{Observations}

LBTI observations-including high-contrast direct imaging and integral field spectroscopy-are scheduled dynamically in queue mode to match observing conditions and project requirements. Most data presented in this work were obtained between 2016 September and 2017 May (LBT observing semesters 2016B and 2017A) as part of the HOSTS survey. In addition, three stars were observed during LBTI commissioning: $\eta$ Crv (2014 February, Defrère et al. 2015), $\epsilon$ Eri (2014 November), and $\beta$ Leo (2015 February, Defrère et al. 2016; P. Hinz et al. 2018, in preparation). A brief $\log$ of the observations is presented in Table 5. All raw and calibrated HOSTS data will be available to the public one year after the observation date through the LBTI Archive (http://lbti. ipac.caltech.edu/).

\subsection{Instrument Description}

The HOSTS observations are carried out using the LBTI (Hinz et al. 2016) at the Large Binocular Telescope (LBT). The instrument combines the light from the two $8.4 \mathrm{~m}$ apertures separated by $14.4 \mathrm{~m}$ (center to center) on a common mount. The two wave fronts are stabilized by adaptive optics (AO) mirrors using two independent, closed-loop adaptive optics subsystems (one for each aperture) with one pyramid wave front sensor each, operating in the $R$ to $I$ band range (Bailey et al. 2014). The infrared light then enters the cryogenically cooled beam combiner. Active optical path delay (OPD) and tip-tilt correction are performed using a closed-loop subsystem with a fringe tracker operating in the $K$ band. Active vibration correction can be performed in the phase loop using telemetry from the Optical Vibration Measurement System (OVMS, Böhm et al. 2016) on the LBT. The mIR light (filter $N^{\prime}$ for the observations presented in this work, $\left.\lambda_{\mathrm{c}}=11.11 \mu \mathrm{m}, \Delta \lambda=2.60 \mu \mathrm{m}\right)$ is then combined in the pupil plane and re-imaged on the Nulling Optimized Mid Infrared Camera (NOMIC; Hoffmann et al. 2014). NOMIC has a pixel scale of $17.9 \mathrm{mas} / \mathrm{pix}$, and the diffraction-limited singleaperture point-spread function (PSF) has an FWHM of 313 mas in the $N^{\prime}$ filter.

\subsection{Observing Strategy}

To obtain a calibrated science observation, an observation of a science target (SCI) is paired with a calibrator observation (CAL). The goal is to obtain for each science target a minimum of three such observations. Two calibrated science observations are typically arranged in a sequence CAL1-SCI-SCI-CAL2, and two such sequences are typically observed per science target. Ideally, four different calibrators are used. The two 
Table 1

Observed Sample as of 2017 June

\begin{tabular}{|c|c|c|c|c|c|c|c|c|c|c|}
\hline $\begin{array}{l}\text { HD } \\
\text { Number }\end{array}$ & Name & $\# \mathrm{SCI}^{\mathrm{a}}$ & Sp. Type & $\begin{array}{c}V \\
(\mathrm{mag})\end{array}$ & $\begin{array}{c}K \\
(\mathrm{mag})\end{array}$ & $\begin{array}{l}N^{\prime b} \\
(\mathrm{Jy})\end{array}$ & $\begin{array}{c}d \\
(\mathrm{pc})\end{array}$ & $\begin{array}{r}\text { EEID }^{\mathrm{c}} \\
\text { (mas) }\end{array}$ & $\begin{array}{c}\mathrm{fIR} / \mathrm{nIR} \\
\text { excess }\end{array}$ & $\begin{array}{l}\text { Excess } \\
\text { references }\end{array}$ \\
\hline \multicolumn{11}{|c|}{ Sensitivity-driven sample ${ }^{\mathrm{d}}$ : } \\
\hline 33111 & $\beta$ Eri & 2 & A3 IV & 2.782 & 2.38 & 3.7 & 27.4 & 248 & $\mathrm{~N} / \mathrm{N}$ & $1,2,3$ \\
\hline 81937 & $23 \mathrm{UMa}$ & 3 & F0 IV & 3.644 & 2.73 & 2.6 & 23.8 & 168 & $\mathrm{~N} / \cdots$ & 4 \\
\hline 95418 & $\beta \mathrm{UMa}$ & 4 & A1 IV & 2.341 & 2.38 & 4.2 & 24.5 & 316 & $\mathrm{Y} / \mathrm{N}$ & 5,6 \\
\hline 97603 & $\delta$ Leo & 4 & A5 IV & 2.549 & 2.26 & 3.9 & 17.9 & 278 & $\mathrm{~N} / \mathrm{N}$ & $1,2,6$ \\
\hline 103287 & $\gamma \mathrm{UMa}$ & 4 & A $0 \mathrm{~V}$ & 2.418 & 2.43 & 3.7 & 25.5 & 308 & $\mathrm{~N} / \cdots$ & $1,2,5$ \\
\hline 106591 & $\delta \mathrm{UMa}$ & 4 & $\mathrm{~A} 2 \mathrm{~V}$ & 3.295 & 3.10 & 2.0 & 24.7 & 199 & $\mathrm{~N} / \mathrm{N}$ & $1,2,6$ \\
\hline 108767 & $\delta \mathrm{Crv}$ & 2 & A0 IV & 2.953 & 3.05 & 2.3 & 26.6 & 251 & $\mathrm{~N} / \mathrm{Y}$ & $1,2,3$ \\
\hline 128167 & $\sigma$ Boo & 3 & $\mathrm{~F} 4 \mathrm{~V}$ & 4.467 & 3.47 & 1.4 & 15.8 & 117 & $\mathrm{Y} / \mathrm{N}^{\mathrm{e}}$ & 1,6 \\
\hline 129502 & $\mu \mathrm{Vir}$ & 3 & $\mathrm{~F} 2 \mathrm{~V}$ & 3.865 & 2.89 & 2.6 & 18.3 & 151 & $\mathrm{~N} / \mathrm{N}$ & 1,2 \\
\hline 172167 & $\alpha$ Lyr & 2 & $\mathrm{~A} 0 \mathrm{~V}$ & 0.074 & 0.01 & 38.6 & 7.68 & 916 & $\mathrm{Y} / \mathrm{Y}$ & 5,7 \\
\hline 187642 & $\alpha \mathrm{Aql}$ & 2 & A7 V & 0.866 & 0.22 & 21.6 & 5.13 & 570 & $\mathrm{~N} / \mathrm{Y}$ & $1,2,6,8$ \\
\hline 203280 & $\alpha$ Cep & 1 & A8 V & 2.456 & 1.85 & 7.0 & 15.0 & 294 & $\mathrm{~N} / \mathrm{Y}$ & $1,2,6,8$ \\
\hline \multicolumn{11}{|c|}{ Sun-like stars sample ${ }^{\mathrm{d}}$ : } \\
\hline 10476 & 107 Psc & 3 & $\mathrm{~K} 1 \mathrm{~V}$ & 5.235 & 3.29 & 2.0 & 7.53 & 90 & $\mathrm{~N} / \mathrm{N}$ & $1,6,9,10$ \\
\hline 16160 & GJ $105 \mathrm{~A}$ & 1 & K3 V & 5.815 & 3.45 & 1.5 & 7.18 & 73 & $\mathrm{~N} / \cdots$ & $1,9,10$ \\
\hline 30652 & 1 Ori & 2 & F6 V & 3.183 & 2.08 & 4.8 & 8.07 & 205 & $\mathrm{~N} / \mathrm{N}$ & $1,6,9,10$ \\
\hline 34411 & $\lambda$ Aur & 2 & G1 V & 4.684 & 3.27 & 1.8 & 12.6 & 105 & $\mathrm{~N} / \cdots$ & 10,11 \\
\hline 48737 & $\xi \mathrm{Gem}$ & 3 & F5 IV-V & 3.336 & 2.13 & 4.3 & 18.0 & 196 & $\cdots / \mathrm{N}$ & 6 \\
\hline 88230 & GJ 380 & 2 & K8 V & 6.598 & 3.21 & 1.9 & 4.87 & 65 & $\mathrm{~N} / \ldots^{\mathrm{f}}$ & 12 \\
\hline 89449 & 40 Leo & 2 & F6 IV-V & 4.777 & 3.65 & 1.1 & 21.4 & 98 & $\mathrm{~N} / \cdots$ & 1,4 \\
\hline 120136 & $\tau$ Boo & 2 & F6 IV & 4.480 & 3.36 & 1.7 & 15.6 & 114 & $\mathrm{~N} / \mathrm{N}$ & $3,10,11$ \\
\hline 126660 & $\theta$ Boo & 3 & F7 V & 4.040 & 2.81 & 3.1 & 14.5 & 147 & $\mathrm{~N} / \cdots$ & $1,9,10$ \\
\hline 141004 & $\lambda$ Ser & 2 & G0 IV-V & 4.413 & 2.98 & 2.4 & 12.1 & 121 & $\mathrm{~N} / \mathrm{N}$ & $1,6,9,13$ \\
\hline 142373 & $\chi$ Her & 3 & G0 V & 4.605 & 3.12 & 2.0 & 15.9 & 111 & $\mathrm{~N} / \mathrm{N}$ & $1,4,6,9$ \\
\hline 142860 & $\gamma$ Ser & 4 & F6 IV & 3.828 & 2.63 & 2.9 & 11.3 & 151 & $\mathrm{~N} / \mathrm{N}$ & $1,6,9,11$ \\
\hline 173667 & $110 \mathrm{Her}$ & 2 & F6 V & 4.202 & 3.03 & 2.2 & 19.2 & 131 & $\mathrm{Y} / \mathrm{Y}$ & 6,12 \\
\hline 185144 & $\sigma$ Dra & 2 & G9 V & 4.664 & 2.83 & 2.7 & 5.76 & 113 & $\mathrm{~N} / \mathrm{N}$ & $6,10,11$ \\
\hline 215648 & $\xi$ Peg A & 3 & F6 V & 4.203 & 2.90 & 2.2 & 16.3 & 132 & $\mathrm{~N} / \mathrm{N}$ & $1,4,9$ \\
\hline \multicolumn{11}{|c|}{ Commissioning targets: } \\
\hline 22049 & $\epsilon$ Eri & 2 & $\mathrm{~K} 2 \mathrm{~V}$ & 3.721 & 1.66 & 7.4 & 3.22 & 172 & $\mathrm{Y} / \mathrm{N}$ & 7,14 \\
\hline 102647 & $\beta$ Leo & 2 & $\mathrm{~A} 3 \mathrm{~V}$ & 2.121 & 1.92 & 6.9 & 11.0 & 336 & $\mathrm{Y} / \mathrm{Y}$ & 5,7 \\
\hline 109085 & $\eta \mathrm{Crv}$ & 3 & $\mathrm{~F} 2 \mathrm{~V}$ & 4.302 & 3.54 & 1.8 & 18.3 & 125 & $\mathrm{Y} / \mathrm{N}$ & 7,15 \\
\hline
\end{tabular}

Notes. Magnitudes are given in the Vega system.

${ }^{\text {a }}$ Number of calibrated science pointings obtained.

${ }^{\mathrm{b}}$ Predicted flux in NOMIC $N^{\prime}$ filter.

${ }^{\mathrm{c}}$ Earth Equivalent Insolation Distance (Section 4.4).

${ }^{\mathrm{d}}$ Section 2.

e Misclassified by Gáspár et al. (2013) as no excess.

${ }^{\mathrm{f}}$ Cold excess (Eiroa et al. 2013) likely background contamination (Gáspár \& Rieke 2014).

References. Spectral type: SIMBAD; $V$ magnitude: Kharchenko et al. (2007); $K$ magnitude: Gezari et al. (1993) and the Lausanne photometric database (http:// obswww.unige.ch/gcpd/); $N$-band flux and EEID: Weinberger et al. (2015); Distance: van Leeuwen (2007); Excess: (1) Gáspár et al. (2013), (2) Thureau et al. (2014), (3) Ertel et al. (2014), (4) Beichman et al. (2006), (5) Su et al. (2006), (6) Absil et al. (2013), (7) Absil et al. (2006) (8) Rieke et al. (2005), (9) Montesinos et al. (2016), (10) Trilling et al. (2008), (11) Lawler et al. (2009), (12) Eiroa et al. (2013), (13) Koerner et al. (2010), (14) Aumann (1985), (15) Aumann (1988).

sequences can be observed independently on different nights and-if needed - can be broken up into the original CAL-SCI or SCI-CAL pairs. Observations of one calibrated science pointing take typically 50 minutes to $1 \mathrm{hr}$.

Observations of SCI and CAL stars are carried out using the same strategy and contain $N_{\text {nods }}$ nodding cycles for background subtraction, a photometric observation, and a sky background observation. Dark frames at the target elevation are taken during each telescope preset to a new star. During the nodding cycles, the beams from both apertures are brought to destructive interference (nulled). The optimum OPD (set point) is determined after each nod by minimizing the residual $N$-band flux on target. This randomizes residual errors in the set-point search and corrects for temporal drifts due to atmospheric water vapor, telescope, and instrumental effects. A nod cycle consists of two on-source nod positions. In each position, $N_{\text {frames }}$ frames with an integration time of typically $45 \mathrm{~ms}$ per frame are obtained. We initially set $N_{\text {nods }}=4$ and $N_{\text {frames }}=1000$, resulting in $2 \times 4 \times 1000=8000$ frames per observation of a star. Based on the experience with the initial reduction of our survey data, we changed these parameters in 2017 January to $N_{\text {nods }}=3$ and $N_{\text {frames }}=2000(2 \times 3 \times 2000=12,000$ frames 
per observation). At the same time, a small, stepwise phase modulation ( $0.2 \mathrm{rad}$ in the $N$ band at a frequency of $\sim 0.4 \mathrm{~Hz}$ ) has been added to break the degeneracy between null depth and residual OPD offset (imperfect destructive interference). ${ }^{15}$ The changes made in the observing strategy reduce the statistical uncertainties by a factor of about two and increase the observing efficiency without introducing any known systematic effects (see Section 4.2 for a discussion of systematics). Thus, all data can be treated and analyzed in a uniform way.

If a significant fraction of the data obtained appears corrupted (e.g., phase loop opened) in our real-time quality control, additional frames or nods are taken. For the photometric observations, the two beams are separated, and the total flux of the source is measured on the two apertures independently, obtaining 500 frames (45 ms each). Finally, the telescope is offset to obtain 1000 sky background frames to be used for sky subtraction of the photometric frames.

\section{Data Reduction and Zodi Measurements}

\subsection{Data Reduction Summary}

Data reduction follows the strategy outlined by Defrère et al. (2016) with minor updates. After a basic reduction of each frame (nod subtraction, bad pixel correction), the source position on the detector is determined for each nod position, and photometry (relative to the total stellar flux, measured on the photometric frames) is performed on each single frame. The raw null depth and its uncertainty are determined using the null self-calibration (NSC), a statistical calibration method originally developed for the Palomar Fiber Nuller experiment (Hanot et al. 2011; Mennesson et al. 2011) and updated for the LBTI (Defrère et al. 2016; Mennesson et al. 2016a). It combines all frames recorded within a given nod.

The measurements from all calibrators in a calibration sequence are combined to determine the instrumental null depth (nulling interferometric transfer function, TF) after correcting for the calibrator diameters. We assume a constant $\mathrm{TF}$ as it is found to be stable within our uncertainties over a calibration sequence: the measurements are first filtered to reject points for which the NSC produced a poor fit to the data $\left(\chi^{2}>5\right.$, less than $\sim 2 \%$ of the data) and obvious outliers in terms of null value or uncertainty (a sign of bad background subtraction or bad data quality, $\lesssim 5 \%$ of the data for a typical night). The remaining measurements from all nod positions are combined using the error weighted mean.

The uncertainty of the final measurement has two main contributions: (1) the uncertainties of the single null measurements obtained from the NSC fit to all data obtained in one nod, and (2) a systematic uncertainty for each nod from imperfect background subtraction. The first contribution can be estimated by combining the NSC uncertainties of the data obtained in each nod to the standard error of the mean. The latter uncertainty is estimated from the scatter (root mean square) of the measurements from all nods, ignoring their NSC uncertainties. The two components are added in quadrature.

The TF is used to calibrate the null measurements of the science target, which are combined using the same strategy and

\footnotetext{
15 An imperfect set point degrades the instrumental null (more stellar flux is transmitted). When observing at a fixed OPD, this effect cannot be distinguished from actual circumstellar emission. Modulating the OPD during the observations eliminates this degeneracy because the OPD-dependent flux is different for the two cases.
}

error estimation as for the calibrator observations. The uncertainty of the TF is added in quadrature to the measurement uncertainty as an additional error term. Observations of the same science target from different nights are combined using the error weighted mean, and its standard error is derived from the uncertainties of the individual measurements. The result is one measurement of the source null (or astrophysical null $N_{\text {as }}$ ) and its uncertainty per target.

\subsection{Measurement Uncertainties}

There are no known, significant systematic uncertainties in our observations that are not already taken into account in the above estimates. Significant general systematics in our observations can be ruled out statistically by analyzing the null distribution of nondetections (Section 5.1). The error from uncertain stellar diameters of our science targets and calibrators is negligible at the LBTI's angular resolution $(<0.01 \%$ null depth error of single calibrators, further reduced by the use of multiple calibrators per science target). The risk of bad calibrators (with companion or circumstellar emission) is minimized by using different calibrators for each science target. No bad calibrators at our sensitivity have been identified in our observations so far. The effects of different pointing directions between science targets and calibrators are mitigated by choosing nearby calibrators (typically within $10^{\circ}$ ), in particular with similar elevation, and are randomized by using several calibrators. We see no significant effect of pointing direction for our selected calibrators. We also see no effects of target brightness in any band. While the magnitude difference between calibrators and corresponding science targets can be as large as a few magnitudes in the visible, all stars observed are by far bright enough for the AO to run at peak performance. In the $K$ band, the calibrators are typically within one magnitude of the corresponding science targets, so the effects on OPD and tip-tilt tracking (still running at peak performance for all stars) are minimal. In the $N$ band, the statistical effects of photon and detector readout noise are dominating in addition to imperfect subtraction of the dominant mIR background and imperfect set point (instrumental null), which are randomized between nods and estimated statistically as described above. For typical observations, the dominant sources of uncertainty are the background subtraction and for faint stars the background photon noise and detector read noise. Both can be considered statistical uncertainties in our observations and data reduction.

\subsection{Dust Distribution and Zodi Definition}

The goal of the HOSTS survey is to constrain the surface density of dust in the HZs of the observed stars. Converting a measured null depth to surface density requires knowledge of - or an assumption on-the radial dust distribution, because we are sensitive to excess originating not only in the $\mathrm{HZ}$ (measured in this work as the Earth Equivalent Insolation Distance, EEID, the distance at which a body receives the same energy density from the star as Earth in the solar system) but also from a range of separations from the star. The radial dust distribution can in principle be constrained from our measurements for detected excesses with high signal-to-noise ratio $(\mathrm{S} / \mathrm{N})$ if the $\mathrm{HZ}$ is at least marginally resolved by the single aperture beam of the instrument or if sufficient auxiliary data are available (e.g., Lebreton et al. 2013; Defrère et al. 2015). In most cases, however, this is not possible. In particular, for 


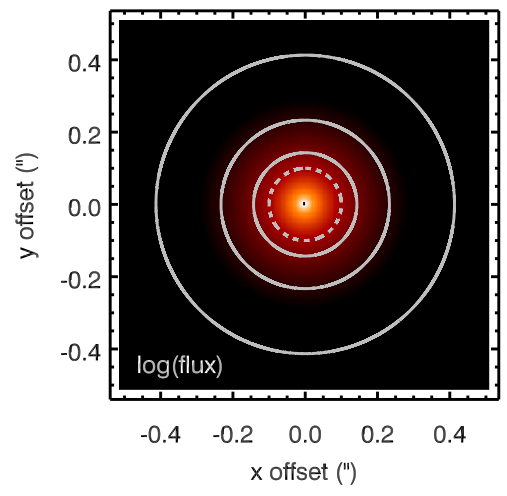

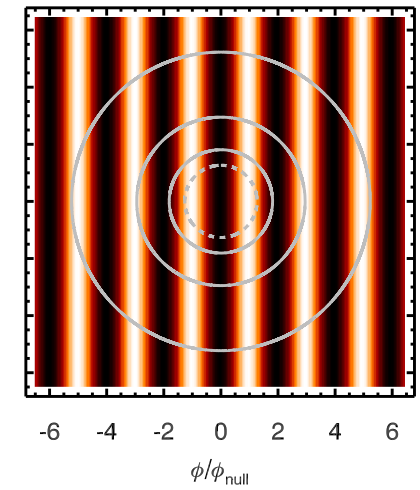
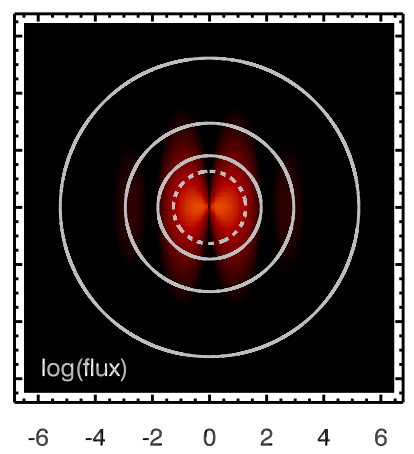

$\phi / \phi_{\text {null }}$
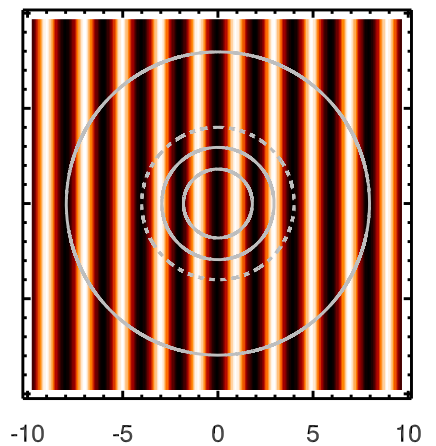

$\phi / \phi_{\text {nul }}$

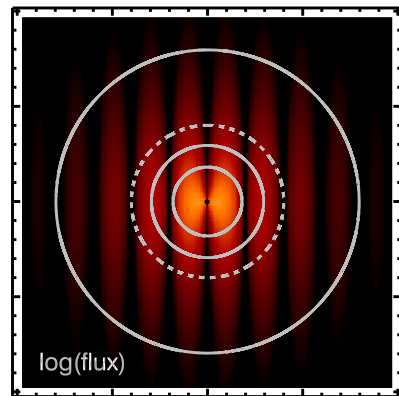

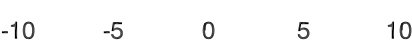

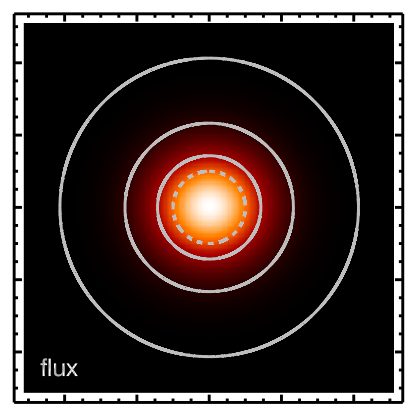

$\begin{array}{lllll}-0.4 & -0.2 & 0.0 & 0.2 & 0.4\end{array}$ $\mathrm{x}$ offset (")

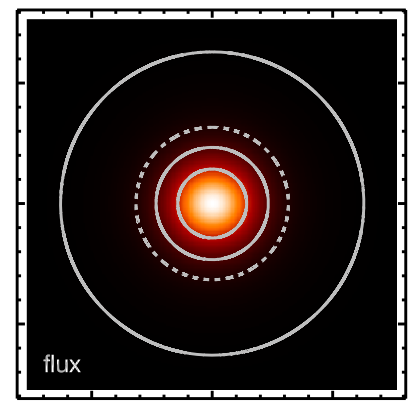

$-0.5$

0.5

x offset (")

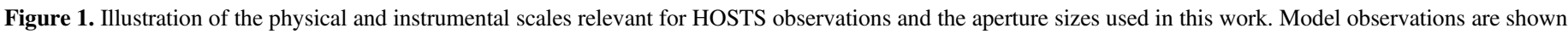

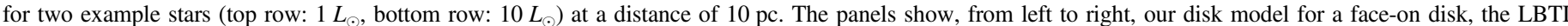

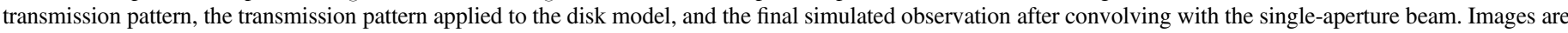

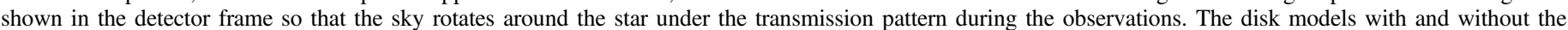

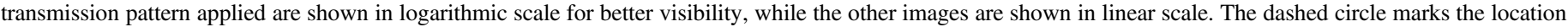
of the EEID. The three solid circles mark from inside out the 8 pix, 13 pix, and conservative apertures.

nondetections where an upper limit on the zodi level must be derived, we need to make an assumption for the radial dust distribution.

We assume a radial dust distribution analogous to that in our solar system with a shallow, inward-increasing dust surface density (solar zodi or SZ model; Kelsall et al. 1998; Kennedy et al. 2015). This model and its application to the HOSTS data have been motivated and discussed in detail by Kennedy et al. (2015), and we only provide a summary here. It is defined by a power-law radial surface density distribution with exponent $\alpha=-0.34$ and inner and outer cutoff radii $r_{\text {in }}$ and $r_{\text {out }}$. We set $r_{\text {in }}$ to the approximate dust-sublimation distance at a blackbody temperature of $1500 \mathrm{~K}$ and $r_{\text {out }}$ to 10 au $\times \sqrt{L / L_{\odot}}$ (scaling the distance with the square root of the stellar luminosity so that a body at this location receives the same energy density as at $10 \mathrm{au}$ from the Sun). The inner edge is then small compared to the inner working angle of the LBTI $(0.5 \times \lambda / B \sim 70$ mas at a baseline $B$ of $14.4 \mathrm{~m}$ ), and the outer edge is large compared to the EEID. Dust inside $r_{\text {in }}$ is not visible to the LBTI, and dust outside $r_{\text {out }}$ is cold, so its emission in the $N$ band is negligible. A power law is the simplest and most general description of the radial dust distribution possible. The assumption of $\alpha=-0.34$, in analogy to the solar system, is a reasonable choice. Simulated images from our model in the $N$ band for face-on disks around stars with luminosities of $1 L_{\odot}$ and $10 L_{\odot}$ at a distance of $10 \mathrm{pc}$ are shown in the left column of Figure 1. We scale this dust distribution so that it has the same vertical geometrical optical depth of $7.12 \times 10^{-8}$ at the EEID as the solar system zodiacal dust at a distance of $1 \mathrm{au}$, which defines our unit of 1 zodi. We compute the expected signal of this model in our LBTI observations by applying the LBTI transmission pattern (Kennedy et al. 2015) and convolving with the single-aperture PSF (the resolution element in ournulled or classical-NOMIC images). This is also illustrated in Figure 1. Fitting the expected signal from this model to the null measurement from a HOSTS observation of a target provides us with a measurement of the $\mathrm{HZ}$ surface density and its uncertainty in units of 1 zodi for each observed star. The free parameters used are the scaling factor and the disk orientation (position angle and inclination) with respect to the hour angle range traced by the LBTI baseline.

It is important to note that the SZ model is a simple geometric model motivated by the dust distribution in our solar system, which is the only available template. It is not necessarily a good representation of a specific exozodiacal dust system. The relative contributions of local dust creation through asteroid collision and comet evaporation, the effects of Poynting-Robertson (PR) and stellar wind drag, the interaction with planets, and the dust removal due to collisions and radiation pressure blowout are unknown for most systems. For example, the surface density of an exozodiacal dust disk has a strong effect on its morphology because collisions will deplete dust more quickly in denser disks (more massive or dust accumulated in resonances with planets), while transport by PR and stellar wind drag will dominate for more tenuous disks (Wyatt 2005; Stark \& Kuchner 2009). This generally leads to a stronger depletion of dust in the inner regions of more massive disks, where the orbital timescale (which is linked to the 
collision timescale together with the disk's surface density; Backman \& Paresce 1993) is shorter.

These caveats are, however, in part mitigated by the design and sensitivity of the LBTI. As can be seen in Figure 1, the location of the first peak of LBTI's transmission pattern is similar to the angular EEID for most of our targets (in particular the Sun-like stars in our sample). Thus, the sensitivity of the LBTI to dust much closer than the EEID is relatively low. Dust much farther away from the star than the EEID is colder and thus fainter in the $N$ band. As a consequence, the region in which we are most sensitive to the dust, the region of interest (the HZ), and the location at which we normalize the dust surface density of our model are very close to each other, minimizing the impact of the uncertain radial dust distribution. In addition, considering collisions and transport of dust grains only, the solar system dust distribution is best suited as a template for less dusty systems that typically result in nondetections in our observations. For our detected, typically more massive disks, more appropriate models can be derived from our data, follow-up observations, and a detailed analysis of auxiliary information about each specific system in future work. In contrast, it is important for the present work to treat all systems in a uniform way.

For detected excesses, we test whether our SZ model is consistent with the null measurements at different aperture sizes (see Section 4.4 for a discussion of the aperture sizes used). While a large range of surface density slopes is possible for most stars due to the typically large uncertainties on the null measurements, all detections are consistent with the SZ model $(\alpha=-0.34)$.

\subsection{Optimum Aperture Size}

The residual source flux in each frame after nulling the central star is measured using aperture photometry and calibrated using an observation of the target star with the two apertures independently (separated on the detector, no interference). The aperture size used is a critical parameter for the result: a small aperture may not cover all of the extended dust emission, including the $\mathrm{HZ}$ of a system, and may thus omit the emission we are most interested in. On the other hand, a larger aperture will produce a larger uncertainty due to photon noise of the sky background, detector readout noise, and differential wave-front aberration effects between the two telescopes that are not captured by NSC. To find the ideal aperture size given the expected flux distribution on our detector, we first simulate images from the SZ model for face-on disks (worst case in terms of flux loss due to limited aperture size), representative of the range of angular EEIDs around our sample stars. Two representative examples are shown in Figure 1.

We find that for this model the majority of the emission is always concentrated in the innermost regions. Even for the largest EEIDs, the main emission is only marginally resolved by the single-aperture NOMIC PSF. This can be explained by the shallow, inward-increasing surface density profile and the fact that dust closer to the star is warmer and thus more luminous. However, our relatively large inner working angle of 70 mas blocks the regions far inside the HZs of most systems (inside one-third of the EEID on average for our sample). Thus, this emission is of little concern for us. Assuming that our measurement uncertainties are dominated by background and read noise (as confirmed by null measurements on calibrators), we find that an aperture radius of 13 pixels ( 233 mas) is very close to the aperture that yields the highest expected $\mathrm{S} / \mathrm{N}$ for the SZ model around all our targets. We thus use this 13 pix aperture as default for our null measurements. This aperture is large enough to encompass the physical size of the EEID directly for all Sun-like stars in our sample and only misses the part of the $\mathrm{HZ}$ emission in the wings of the singleaperture NOMIC PSF.

Because the actual radial dust distribution around our targets is not known, we use two more aperture sizes of potential interest: first, an aperture radius of one half width at half maximum of the NOMIC PSF - quantized to an 8 pix aperture of 143 mas - covers one angular resolution element, which is relevant in case the emission is very compact. This could, for example, be the case for very hot dust, for which the LBTI could see the Rayleigh-Jeans tail of the emission if it is located outside LBTI's central dark fringe.

Second, we use an aperture large enough to miss only negligible amounts of any significant $N$-band emission from the system. We find that an aperture with a radius of 1 EEID plus 1 FWHM (313 mas) of the single-aperture NOMIC PSF achieves this goal. While the flux lost for any realistic dust distribution will be negligible, this large aperture size will be particularly affected by noise. With this aperture we are thus the least sensitive, but it is conservative in terms of neglected flux (conservative aperture).

Null measurements for the 8 pix, 13 pix, and conservative apertures are provided in this work. We limit ourselves to these three apertures for a general and efficient analysis of the whole sample. For detailed analyses of specific objects, a larger range of apertures is used to extract as much information as possible from the data (Defrère et al. 2015, 2016; P. Hinz et al. 2018 , in preparation).

In all cases, the inner edge of the background annulus used for the photometry is set to 1 EEID +1 FWHM. Its width is chosen to cover an area of the same size on the detector as the photometric aperture. The typically large inner edge of the background annulus compared to the 8 and 13 pix photometric apertures avoids the $\mathrm{HZ}$ and interior regions, where significant $N$-band emission might be present. At the same time, a background annulus as close to the photometric aperture as possible minimizes the error introduced by inhomogeneity of the background across the detector.

We make two exceptions for the commissioning targets $\eta \mathrm{Crv}$ and $\beta$ Leo, for which null measurements were already available prior to this work (Defrère et al. 2015, 2016; P. Hinz et al. 2018, in preparation). These data had to be reduced very carefully and with more human intervention due to the less standardized observing strategy and data format and limited data quality. This resulted in better null accuracy than our standardized data reduction can provide for these observations. In order to use the most accurate measurements and to avoid having different but fully consistent measurements in the literature, we use for these stars an aperture size of 10 pix instead of 13 pix. Among the apertures for which the null depths were measured previously at high precision, this aperture is the closest to the optimum size for these stars. Instead of the conservative aperture, we also use the closest aperture size measured. We note that these exceptions are of no consequence for the conclusions of this paper, since the excess detections for these two stars are not in question and their zodi levels are not used in what follows. Additional exceptions are necessary for Vega ( $\alpha$ Lyr) and Altair ( $\alpha$ Aql). For both stars, 
Table 2

Null Measurements and Zodi Levels

\begin{tabular}{|c|c|c|c|c|c|c|c|c|c|c|c|c|c|}
\hline \multicolumn{2}{|c|}{ Aperture $\rightarrow$} & \multicolumn{2}{|c|}{8 pix } & \multicolumn{2}{|c|}{13 pix } & \multicolumn{3}{|c|}{ Conservative } & \multirow[b]{2}{*}{$\begin{array}{l}\text { Aperture } \\
\text { for zodi }\end{array}$} & \multirow[b]{2}{*}{$\begin{array}{c}N_{\mathrm{as}, 1} \\
(\%)\end{array}$} & \multirow[b]{2}{*}{$\begin{array}{c}z \\
\text { (zodi) }\end{array}$} & \multirow[b]{2}{*}{$\begin{array}{c}\sigma_{z} \\
\text { (zodi) }\end{array}$} & \multirow[b]{2}{*}{$z / \sigma_{z}$} \\
\hline $\begin{array}{l}\text { HD } \\
\text { number }\end{array}$ & Name & $\begin{array}{l}N_{\mathrm{as}} \\
(\%)\end{array}$ & $\begin{array}{c}\sigma_{N} \\
(\%)\end{array}$ & $\begin{array}{l}N_{\mathrm{as}} \\
(\%)\end{array}$ & $\begin{array}{c}\sigma_{N} \\
(\%)\end{array}$ & $\begin{array}{c}r_{\mathrm{ap}} \\
(\mathrm{pix})\end{array}$ & $\begin{array}{l}N_{\text {as }} \\
(\%)\end{array}$ & $\begin{array}{c}\sigma_{N} \\
(\%)\end{array}$ & & & & & \\
\hline \multicolumn{14}{|c|}{ Sensitivity-driven sample (Spectral types A to F5): } \\
\hline 33111 & $\beta$ Eri & -0.004 & 0.110 & 0.168 & 0.119 & 18 & 0.372 & 0.176 & 13 pix & $4.45 \times 10^{-3}$ & 37.8 & 26.7 & 1.4 \\
\hline 81937 & $23 \mathrm{UMa}$ & 0.003 & 0.073 & 0.013 & 0.092 & 25 & 0.008 & 0.179 & 13 pix & $2.60 \times 10^{-3}$ & 4.9 & 35.3 & 0.1 \\
\hline 95418 & $\beta \mathbf{U M a}$ & 0.920 & 0.055 & 1.019 & 0.060 & 33 & 1.655 & 0.102 & 13 pix & $6.49 \times 10^{-3}$ & 156.9 & 9.2 & 17.1 \\
\hline 97603 & $\delta$ Leo & 0.028 & 0.051 & 0.033 & 0.055 & 32 & -0.013 & 0.143 & 13 pix & $5.49 \times 10^{-3}$ & 6.1 & 10.0 & 0.6 \\
\hline 103287 & $\gamma \mathrm{UMa}$ & -0.037 & 0.033 & 0.003 & 0.031 & 35 & 0.083 & 0.080 & 13 pix & $7.02 \times 10^{-3}$ & 0.4 & 4.4 & 0.1 \\
\hline 106591 & $\delta$ UMa & 0.366 & 0.094 & 0.436 & 0.109 & 28 & 0.523 & 0.184 & 13 pix & $5.12 \times 10^{-3}$ & 85.2 & 21.2 & 4.0 \\
\hline 108767 & $\delta \mathrm{Crv}$ & -0.333 & 0.131 & -0.243 & 0.199 & 26 & 0.933 & 0.365 & 13 pix & $7.45 \times 10^{-3}$ & -32.6 & 26.8 & -1.2 \\
\hline 128167 & $\sigma$ Boo & -0.019 & 0.096 & -0.006 & 0.118 & 22 & 0.417 & 0.252 & 13 pix & $2.10 \times 10^{-3}$ & -2.7 & 56.0 & -0.1 \\
\hline 129502 & $\mu \mathrm{Vir}$ & -0.006 & 0.092 & 0.183 & 0.110 & 25 & 0.192 & 0.198 & 13 pix & $1.95 \times 10^{-3}$ & 93.8 & 56.7 & 1.7 \\
\hline 172167 & $\alpha \mathrm{Lyr}$ & -0.037 & 0.050 & 0.022 & 0.061 & $37^{\mathrm{a}}$ & 0.240 & 0.150 & 13 pix & $4.62 \times 10^{-3}$ & 4.7 & 13.1 & 0.4 \\
\hline 187642 & $\alpha \mathrm{Aql}$ & -0.032 & 0.166 & 0.217 & 0.192 & $47^{\mathrm{a}}$ & -0.995 & 0.356 & 13 pix & $3.84 \times 10^{-3}$ & 56.5 & 50.1 & 1.1 \\
\hline 203280 & $\alpha$ Cep & -0.301 & 0.376 & -0.233 & 0.182 & 18 & -0.075 & 0.266 & 13 pix & $3.36 \times 10^{-3}$ & -69.4 & 54.3 & -1.3 \\
\hline \multicolumn{14}{|c|}{ Sun-like stars sample (Spectral types F6 to K8): } \\
\hline 10476 & 107 Psc & -0.028 & 0.083 & -0.027 & 0.122 & 21 & 0.154 & 0.181 & 13 pix & $6.36 \times 10^{-4}$ & -42 & 192 & -0.2 \\
\hline 16160 & GJ $105 \mathrm{~A}$ & 0.228 & 0.232 & -0.227 & 0.239 & 18 & 0.538 & 0.363 & 13 pix & $4.49 \times 10^{-4}$ & -506 & 533 & -1.0 \\
\hline 30652 & 1 Ori & 0.098 & 0.183 & 0.347 & 0.217 & 28 & 0.209 & 0.351 & 13 pix & $2.27 \times 10^{-3}$ & 152.5 & 95.3 & 1.6 \\
\hline 34411 & $\lambda$ Aur & -0.210 & 0.095 & -0.108 & 0.079 & 22 & 0.041 & 0.136 & 13 pix & $1.16 \times 10^{-3}$ & -93.3 & 68.3 & -1.4 \\
\hline 48737 & $\xi \mathrm{Gem}$ & 0.048 & 0.099 & 0.124 & 0.098 & 27 & 0.057 & 0.229 & 13 pix & $2.20 \times 10^{-3}$ & 56.4 & 44.6 & 1.3 \\
\hline 88230 & GJ 380 & -0.111 & 0.059 & -0.077 & 0.056 & 20 & -0.189 & 0.087 & 13 pix & $2.59 \times 10^{-4}$ & -299 & 217 & -1.4 \\
\hline 89449 & 40 Leo & 0.238 & 0.263 & -0.018 & 0.290 & 21 & 1.278 & 0.578 & 13 pix & $1.51 \times 10^{-3}$ & -12 & 192 & -0.1 \\
\hline 120136 & $\tau$ Boo & -0.046 & 0.191 & -0.313 & 0.148 & 22 & 0.343 & 0.456 & 13 pix & $1.50 \times 10^{-3}$ & -208.3 & 98.7 & -2.1 \\
\hline 126660 & $\theta$ Boo & 0.276 & 0.082 & 0.362 & 0.085 & 24 & 0.362 & 0.103 & 13 pix & $1.55 \times 10^{-3}$ & 234.0 & 54.8 & 4.3 \\
\hline 141004 & $\lambda$ Ser & 0.015 & 0.036 & 0.025 & 0.047 & 23 & -0.107 & 0.117 & 13 pix & $1.20 \times 10^{-3}$ & 21.0 & 39.2 & 0.5 \\
\hline 142373 & $\chi$ Her & -0.063 & 0.052 & 0.112 & 0.061 & 22 & 0.071 & 0.083 & 13 pix & $1.13 \times 10^{-3}$ & 99.7 & 53.7 & 1.9 \\
\hline 142860 & $\gamma$ Ser & 0.037 & 0.044 & -0.009 & 0.058 & 25 & 0.023 & 0.079 & 13 pix & $1.78 \times 10^{-3}$ & -4.9 & 32.4 & -0.2 \\
\hline 173667 & 110 Her & 0.126 & 0.096 & 0.101 & 0.115 & 24 & 0.561 & 0.157 & cons. & $1.98 \times 10^{-3}$ & 283.3 & 79.0 & 3.6 \\
\hline 185144 & $\sigma$ Dra & 0.027 & 0.052 & -0.075 & 0.071 & 22 & -0.096 & 0.096 & 13 pix & $8.82 \times 10^{-4}$ & -85.4 & 80.7 & -1.1 \\
\hline 215648 & $\xi \operatorname{Peg} \mathrm{A}$ & 0.154 & 0.121 & 0.226 & 0.167 & 23 & 0.198 & 0.214 & 13 pix & $1.61 \times 10^{-3}$ & 140 & 103 & 1.4 \\
\hline \multicolumn{14}{|c|}{ Commissioning targets: } \\
\hline 22049 & $\epsilon$ Eri & 0.037 & 0.147 & 0.206 & 0.142 & 27 & 0.901 & 0.269 & cons. & $1.24 \times 10^{-3}$ & 724 & 216 & 3.4 \\
\hline 102647 & $\boldsymbol{\beta}$ Leo & 0.470 & 0.050 & $0.420^{b}$ & 0.054 & 32 & 1.160 & 0.333 & 8 pix & $4.00 \times 10^{-3}$ & 117.4 & 12.5 & 9.4 \\
\hline 109085 & $\eta \mathrm{Crv}$ & 4.410 & 0.350 & $4.580^{b}$ & 0.460 & 24 & 4.710 & 0.890 & 8 pix & $1.67 \times 10^{-3}$ & 2649 & 210 & 12.6 \\
\hline
\end{tabular}

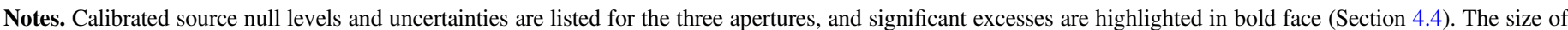

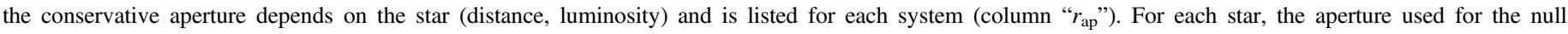

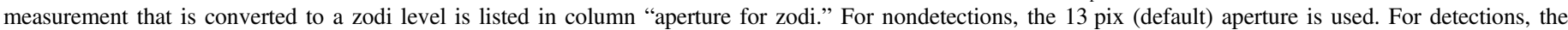
aperture that produces the highest $\mathrm{S} / \mathrm{N}$ is used.

a The aperture used for these targets is smaller than the actual conservative aperture due to limitations of the usable detector area (Section 4.4).

b An aperture of 10 pix instead of 13 pix is used for these stars (Section 4.4).

the conservative aperture plus the corresponding background annulus do not fit into the usable detector area (one stripe with a size of 128 pix $=2$ ". 3 ). Thus, the conservative aperture and background annulus were set to the largest possible size (Table 2). For Altair the difference is minor, but for Vega the largest aperture radius used is only about two-thirds of the EEID and only about one-half of the size of the corresponding conservative aperture. Similar to $\eta \mathrm{Crv}$ and $\beta \mathrm{Leo}$, the exception for Vega is of little consequence for the conclusions of this paper.

Correction factors for the flux lost to a finite aperture size and null-to-zodi conversion factors for all stars observed are derived from our SZ model for the apertures used. For nondetections, the null measurements and uncertainties measured using the 13 pix aperture are converted to zodi levels and uncertainties. In case of a detection, we use the measurement derived from the aperture that produced the highest $\mathrm{S} / \mathrm{N}$. We find that the uncertainties on the aperture corrections and nullto-zodi conversions caused by the unknown disk orientation are negligible compared to the measurement uncertainties for all our targets.

\section{Results}

\subsection{Excess Significance and Detection Threshold}

The resulting measurements and uncertainties on the source null levels $\left(N_{\mathrm{as}}, \sigma_{N}\right)$ and derived zodi levels are listed in Table 2 for all three aperture sizes. The distributions of the excess significance $N_{\text {as }} / \sigma_{N}$ and of the uncertainties are plotted in Figure 2. For the 8 pix aperture, we can see that the excess significance distribution $N_{\text {as }} / \sigma_{N}$ follows a normal distribution for $-3<N_{\mathrm{as}} / \sigma_{N}<3$ with the addition of several 

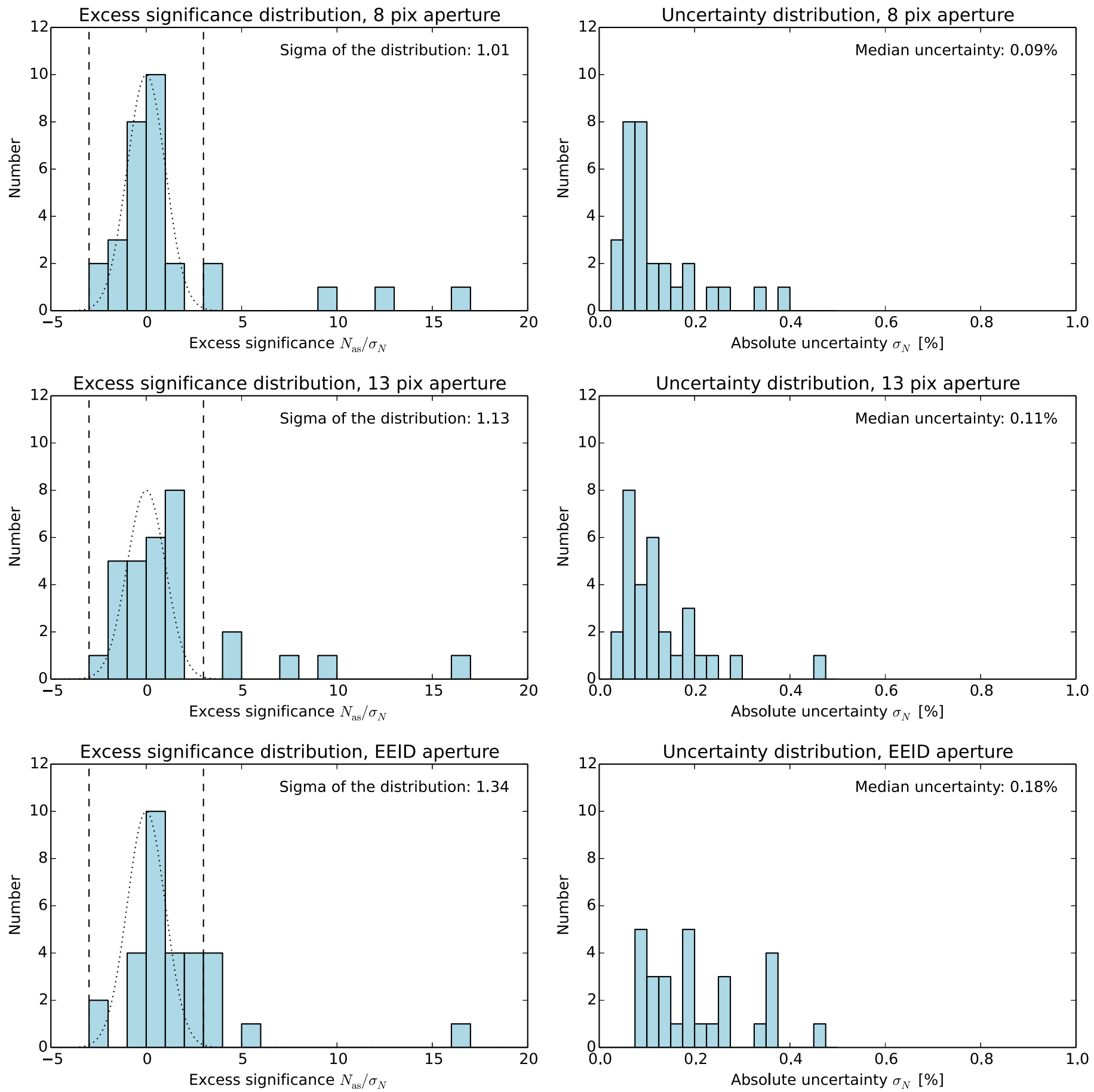

Figure 2. Distribution of excess significance $N_{\mathrm{as}} / \sigma_{N}$ (left) and uncertainties $\sigma_{N}$ (right) for our targets when measured using the three aperture sizes described in Section 4.4 (top to bottom: 8 pix, 13 pix, and conservative). The two vertical, dashed lines in the excess significance distribution plots mark the $\pm 3 \sigma$ boundaries based on our uncertainty estimates. The standard deviation of the distribution is computed from nondetections only $\left(-3<N_{\text {as }} / \sigma_{N}<3\right)$. The dotted line represents a Gaussian with a standard deviation of one (normal distribution) scaled to the peak of the histogram and is used only to guide the eye.

measurements at $N_{\mathrm{as}} / \sigma_{N}>3$. The distribution of the measurement uncertainty is also well behaved, with the majority of the targets having uncertainties close to the median of $0.09 \%$ (absolute uncertainty of the null measurement, expressed as a fraction of the total stellar flux) and a tail of measurements at larger uncertainties. This tail can in part be explained by the fact that the observations for a fraction of our stars are still incomplete and the measurements of the null depth of these stars are less precise.
These results validate our strategies for data reduction, null measurement, and error estimation. We thus apply a $3 \sigma$ threshold to identify significant excesses in our sample. We detect significant excesses around $\beta \mathrm{UMa}, \delta \mathrm{UMa}$, and $\theta$ Boo in addition to the two previously reported excesses around $\eta \mathrm{Crv}$ (Defrère et al. 2015) and $\beta$ Leo (Defrère et al. 2016; P. Hinz et al. 2018, in preparation).

For the two larger apertures, the distribution of $N_{\mathrm{as}} / \sigma_{N}$ is similar but skewed toward positive values. Except for the 
different aperture size, the approach to derive the source null levels and uncertainties is identical for all apertures. We see no reason why an instrumental, observational, or data reduction bias should be present when using a larger aperture but not when using the 8 pix aperture. Effects such as an imperfect tiptilt correction (thus imperfect overlap of the PSFs from both apertures, resulting in less deep instrumental nulls) that could have stronger effects at larger separation from the center of the PSFs are expected to be present for both science targets and calibrators. Moreover, if at all significant, the effect is expected to be more pronounced on our calibrators, which are often slightly fainter in the $R$ to $I$ band than our science targets. This would skew the distribution toward negative excesses rather than positive ones. As expected, the uncertainties increase with increasing aperture size. While the distribution for the 13 pix aperture is still well behaved, it is more scattered for the conservative aperture. This is due to the variable size of the conservative apertures for each star and the size-dependent noise behavior.

Given this discussion, the shapes of the excess significance distributions for larger aperture sizes might indicate a potential population of faint, extended excesses below our formal $3 \sigma$ detection threshold. These would then not be obvious using the 8 pix aperture, because the majority of the emission would be located outside this small aperture. We note, however, that the changes of the histograms with aperture size are not significant and can also be explained by statistical fluctuations due to our small sample size. As a consequence, we also employ a $3 \sigma$ detection threshold to identify significant excesses among the null measurements with the larger apertures. For the 13 pix aperture, we recover the three new detections made with the 8 pix aperture at slightly larger significance, as expected. Using the conservative aperture, we find two more detections around 110 Her and $\epsilon$ Eri. The fact that no excess for these two stars was found using the smaller apertures might suggest that the dust is concentrated close to the HZ, with a clearing closer in. However, given the large error bars, all measurements of both targets are still consistent with the SZ model, which also predicts a flux increase with aperture size. We claim detections for both stars, albeit at a significance of $\sim 3.5 \sigma$ only in both cases, so these probable detections deserve confirmation. The excess for $\delta \mathrm{UMa}$ with the conservative aperture is not significant $(2.8 \sigma)$, due to the increased uncertainty.

\subsection{Notes on Specific Targets}

$\epsilon$ Eri (Ran, HD 22049, HIP 16537) is a very nearby (3.2 pc van Leeuwen 2007), chromospherically active K2 V star that hosts one of the first debris disks discovered by IRAS (Gillett 1986). The disk has been extensively studied since. The age of the star is not well known but appears from gyrochronology and activity calibrations to be 400-800 Myr (Barnes 2007; Mamajek \& Hillenbrand 2008). The substantial cool dust (fractional luminosity $L_{\mathrm{IR}} / L_{*} \sim 4 \times 10^{-5}, T \sim 50$ $K$, Greaves et al. 2014) was first spatially resolved by submillimeter imaging (Greaves et al. 1998) as a potentially clumpy ring at $\sim 65$ au with a width of $<14$ au and a slight offset from the star with a low eccentricity (Backman et al. 2009; Lestrade \& Thilliez 2015; MacGregor et al. 2015; Chavez-Dagostino et al. 2016; Booth et al. 2017). The clumpy structure has, however, been debated and could only be reproduced in one of several follow-up observations (Lestrade \& Thilliez 2015), which suggests a contribution from background contamination. Excess emission interior to the well-known ring has been detected by a combination of direct imaging, mIR spectroscopy, and spectral energy distribution (SED) modeling (Backman et al. 2009; Reidemeister et al. 2011; Greaves et al. 2014) and was resolved with a size of $\sim 12 \mathrm{au}$ ( $\mathrm{Su}$ et al. 2017). Additional millimeter-wave emission at the location of the star is likely due to stellar chromospheric emission (MacGregor et al. 2015; Booth et al. 2017). We detect significant $N$-band excess emission from this star only with the conservative aperture $\left(N_{\mathrm{as}}=[0.90 \pm\right.$ $0.271 \%$ ). This might suggest some inner clearing of dust, but our limited-quality commissioning data are still consistent with the SZ model for all apertures. The star was observed with $\mathrm{KIN}$, in principle at sufficient sensitivity to detect the excess we find (Mennesson et al. 2014). However, the use of a pinhole limited the field of view to an FWHM of 400 mas, more than a factor of two smaller than our conservative aperture for this system. KIN was thus unable to detect the excess suggested by our LBTI observations. If the strong excess is confirmed by higher quality survey data, a detailed analysis of the radial dust distribution will allow us to put strong constraints on its origin. Our data can be compared to detailed predictions on the warm dust distribution for various formation and evolution scenarios available in the literature (Moran et al. 2004; Brogi et al. 2009; Reidemeister et al. 2011). Interestingly, the radius of the conservative aperture is close to the location of a dust clump that was tentatively detected through $Q$-band imaging (S. Ertel et al. 2018, in preparation). This could indicate local dust production in the known asteroid belt and potential shepherding by a planet interior to the belt, which could also be creating the clump. There is a long history of planet claims for $\epsilon$ Eri, but radial velocity detection is complicated by jitter induced by stellar activity. The existence of the planet claimed by Hatzes et al. (2000) and Benedict et al. (2006) has been debated in the literature (Anglada-Escudé \& Butler 2012; Howard \& Fulton 2016); it is possible that a planet of period 6.8-7.3 years and mass $0.6-1.55 M_{\text {Jup }}$ does orbit the star. Attempts to infer the presence of outer planets based on the ring structure are problematic due to the uncertain nature of the intrinsic disk morphology.

110 Her (HD 173667, HIP 92043) is a well-studied F6 V star at 19.2 pc. It has solar or slightly subsolar metallicity and an age of 3 Gyr (compilation by Trilling et al. 2008). A faint far-infrared (fIR) excess was first suggested by Eiroa et al. (2013). An unresolved 3-4 $\sigma$ excess was confirmed by the focused analysis of this system by Marshall et al. (2013) and attributed to a regular Kuiper Belt-like debris disk, although the low-significance detection may be questioned (Gáspár et al. 2013). In addition, a marginal, extended excess emission was found by both Eiroa et al. (2013) and Marshall et al. (2013). It may be attributed to the presence of a very cold disk (Eiroa et al. 2011; Krivov et al. 2013), but it has been shown that background contamination is a likely alternative (Marshall et al. 2013; Gáspár \& Rieke 2014). The excess was not detected independently by Spitzer, but its reality at $70 \mu \mathrm{m}$ is supported at the $2.3 \sigma$ level (Gáspár et al. 2013). No excess was found at 8-30 $\mu \mathrm{m}$ with Spitzer/IRS (Beichman et al. 2006; Lawler et al. 2009). We here follow the focused analysis by Marshall et al. (2013) and consider the star a debris disk host star, but we urge caution with this conclusion due to the low-significance detection. Absil et al. (2013) and Nuñez et al. (2017) find a significant $K$-band excess using CHARA/FLUOR interferometric observations. Similar to $\epsilon$ Eri, we detect an $\mathrm{mIR}$ 
excess around $110 \mathrm{Her}$ only in the conservative aperture $\left(N_{\mathrm{as}}=\right.$ $[0.561 \pm 0.157] \%)$, but the measurements with all apertures are also consistent within the uncertainties with the $\mathrm{ZS}$ model. The absence of a massive, cold dust belt puts into question whether the $\mathrm{HZ}$ dust can be produced in a (tenuous) outer disk and migrate inward due to PR drag, as was suggested by Kennedy \& Piette (2015). It has also been shown that the nIR excess cannot be explained by PR drag, even in the presence of a massive, cold dust belt (Wyatt et al. 2007; van Lieshout et al. 2014). Instead, both the nIR and mIR excess in this system could be produced by evaporating comets that would not necessarily originate in a detectable Kuiper Belt (Bonsor et al. 2014; Faramaz et al. 2017). The star has no detected radial velocity planet at the level of a few $10 \mathrm{~m} \mathrm{~s}^{-1}$ (Fischer et al. 2014; Howard \& Fulton 2016).

$\beta$ UMa (Merak, HD 95418, HIP 53910) is an early A-type star at a distance of $24.5 \mathrm{pc}$ (van Leeuwen 2007). Excesses are consistently detected at wavelengths $>24 \mu \mathrm{m}$ with Spitzer/MIPS at 24 and $70 \mu \mathrm{m}$, and with Spitzer/IRS between 30 and $34 \mu \mathrm{m}$ (Chen et al. 2006). The circumstellar emission was also resolved by Herschel/PACS at 70 and $100 \mu \mathrm{m}$ (Booth et al. 2013), with a very clean fit to the data obtained by a narrow dust ring at about 43 au and seen close to edge-on (inclination $\sim 84^{\circ}$, position angle $114^{\circ}$, though with considerable uncertainties). A warm disk component was first resolved at $11.2 \mu \mathrm{m}$ (Moerchen et al. 2010) with an inclination and position angle consistent with that of the outer disk. Its excess flux was first quantified with KIN (Mennesson et al. 2014). We redetect the excess at a very high significance of $[0.920 \pm 0.055] \%, \quad[1.019 \pm 0.060] \%$, and $[1.66 \pm 0.10] \%$ in the 8 pix, 13 pix, and conservative apertures, respectively. A $3 \sigma$ upper limit of $0.43 \%$ was put on the hot excess around this star in the $K$ band by Absil et al. (2013). An origin of the HZ dust in the massive outer disk seems plausible for this system. The combined data from KIN and LBTI at different inner working angles, fields of view/aperture sizes, and baseline orientations combined with the nIR and mIR to fIR spectrophotometric data and resolved images constitute a rich data set, and detailed modeling of the system by our team is ongoing.

$\beta$ Leo (Denebola, HD 102647, HIP 57632) is an A3 V star with a luminosity of $15 L_{\odot}$, distance of $11.0 \mathrm{pc}$, and isochronal estimates of its age ranging from $50 \mathrm{Myr}$ (Chen et al. 2006) to $100 \mathrm{Myr}$ (Vican 2012). It has been proposed to be a member of the $\sim 40$-Myr-old Argus moving group (Zuckerman et al. 2011). $\beta$ Leo has been identified as a probable $\delta$ Scuti variable (Frolov 1970). As a nearby young star, it is a prime target for direct imaging campaigns, but they have been thus far unsuccessful (Meshkat et al. 2015; Durkan et al. 2016). The dust around $\beta$ Leo has been studied extensively, with infrared excesses previously reported from cold dust using IRAS (Aumann 1985) and Spitzer (Chen et al. 2006; Su et al. 2006), from warm dust at $8.5 \mu \mathrm{m}$ using KIN (Mennesson et al. 2014), and from hot dust using the CHARA/FLUOR interferometric observations in the $K$ band (Akeson et al. 2009; Absil et al. 2013). Direct imaging at 100 and $160 \mu \mathrm{m}$ with Herschel resolved a roughly face-on, cold dust disk extending to $\sim 40$ au (Matthews et al. 2010). The resolved disk cannot produce the observed flux at short wavelengths, leading Stock et al. (2010) to produce a two-component dust model with planetesimal belts from 2-3 au to 5-55 au, and Churcher et al. (2011) to suggest a three-component model with belts at 2 au, $9 \mathrm{au}$, and 30-70 au. The LBTI detection of an excess $\left(N_{\mathrm{as}}=\right.$ $[0.470 \pm 0.050] \%$ in the 8 pix aperture) and its aperture size dependence are being studied in detail by P. Hinz et al. (2018, in preparation), finding that the measurement is consistent with dust spiraling in from the outer belt due to PR drag. The origin of the hot dust remains unclear in this scenario but could be related to comet delivery from the outer disk.

$\delta$ UMa (Megrez, HD 106591, HIP 59774) is an A2 V star at a distance of $24.7 \mathrm{pc}$. The star is a rapid rotator, which has been taken into account for its age estimate of $400 \mathrm{Myr}$ (Jones et al. 2015). An infrared excess at both 24 and $70 \mu \mathrm{m}$ was identified by Su et al. (2006), but only at $\sim 4 \sigma$ in both bands. A reanalysis of the Spitzer data and newer Herschel data disproves the excess (Gáspár et al. 2013; Thureau et al. 2014). Our excess detection $\left(N_{\text {as }}=[0.436 \pm 0.109] \%\right.$ in the 13 pix aperture) is particularly interesting because this is a star without any previously known dust (despite sensitive fIR observations) but with an $\mathrm{mIR}$ interferometric detection of exozodiacal dust.

$\eta$ Crv (HD 109085, HIP 61174) is an early-type star (spectral type $\mathrm{F} 2 \mathrm{~V}$ ) at a distance of $18.3 \mathrm{pc}$. It has an age of 1-2 Gyr (e.g., Ibukiyama \& Arimoto 2002; Mallik et al. 2003; Vican 2012). The star is well known for its prominent debris disk at $152 \mathrm{au}$ and massive warm dust system (Wyatt et al. 2005; Beichman et al. 2006; Chen et al. 2006; Lisse et al. 2012; Duchêne et al. 2014; Marino et al. 2017). The latter has been studied in detail by Lebreton et al. (2016) using midinfrared interferometry from KIN (Smith et al. 2009; MillanGabet et al. 2011) and LBTI (Defrère et al. 2015) and available $\mathrm{mIR}$ and fIR spectroscopic and photometric data. We list for this star the source null level of $N_{\text {as }}=[4.41 \pm 0.35] \%$ published by Defrère et al. (2015), which has been measured for the 8 pix aperture and was found not to depend significantly on aperture size. The excess measured by the LBTI has been found to be small compared to that expected from earlier spectroscopic and photometric observations. This, together with the aperture-independent excess, led to the conclusion that the majority of the emission must be rather compact, located at a separation of $0.5-1.0 \mathrm{au}$ from the star, close to the inner working angle of the LBTI. Such large amounts of warm dust close to a gigayear-old star must be transient (Wyatt et al. 2007), suggesting that it was produced in a recent, catastrophic collision. Surprisingly, no nIR excess was detected around this star with a $3 \sigma$ upper limit of $1.5 \%$ (Absil et al. 2013).

$\theta$ Boo (HD 126660, HIP 70497) is an F7 V star at $14.5 \mathrm{pc}$, with a luminosity of $4 L_{\odot}$, of nearly solar abundance and age of 1-2 Gyr (Montesinos et al. 2016). It has been searched for infrared excess with both Spitzer (Trilling et al. 2008) and Herschel (Montesinos et al. 2016), with no detection of excess at wavelengths up to $160 \mu \mathrm{m}$. The star has not been searched for the presence of hot dust using nIR interferometry. We find an excess of $N_{\text {as }}=[0.362 \pm 0.085] \%$ in the 13 pix aperture, making this star the second one-after $\delta \mathrm{UMa}-$ in our sample for which we find a detection without previously known fIR excess.

$\alpha$ Lyr (Vega, HD 172167, HIP 91262) has, of course, the prototypical debris disk (Aumann et al. 1984). The star is conventionally classified as type $\mathrm{A} 0 \mathrm{~V}$. However, it is very rapidly rotating and seen pole-on, and hence has a large temperature gradient $(\sim 2000 \mathrm{~K})$ from its pole to equator (Gulliver et al. 1994; Aufdenberg et al. 2006; Peterson et al. 2006). Vega is at a distance of $7.68 \pm 0.02 \mathrm{pc}$. The rapid rotation complicates conventional estimates for its age 
(Peterson et al. 2006; Yoon et al. 2008); correcting for its effects, the age is estimated at $455 \pm 13 \mathrm{Myr}$ (Yoon et al. 2010). The Vega debris disk is complex. It has an excess most likely due to very hot dust in the near-infrared, at a level of $1.29 \pm 0.19 \%$ of its photosphere at $2.12 \mu \mathrm{m}$, within a field of view of $\sim 7.8 \mathrm{au}$, and detected at a similar level but at lower significance, that is, $1.23 \pm 0.45 \%$, at $1.65 \mu \mathrm{m}$ (Defrère et al. 2011; Absil et al. 2013). Previous nulling interferometry at $10 \mu \mathrm{m}$ (Liu et al. 2004) established an upper limit requiring the spectrum of this excess to be as blue as Rayleigh-Jeans or its source to lie within 0.8 au of the star. KIN measurements by Mennesson et al. (2014) rule out red excess generated between 0.05 and 1.5 au with a $3 \sigma$ upper limit of about $1.25 \%$ of the photosphere $(\sim 2.5$ times the source null). The LBTI upper limits of $0.12 \%, 0.2 \%$, and $0.7 \%$ source null in an aperture of $1.1,1.8$, and 5.1 au (8 pix, 13 pix, and conservative aperture), respectively, with an inner working angle of $0.5 \mathrm{au}$ further strengthen the constraints on the dust location and emission. Considering all arguments, the hot dust is most likely located inside LBTI's inner working angle, and the KIN measurement still provides the strongest constraint on its mIR emission. This is plausible as the blackbody equilibrium temperature at $0.5 \mathrm{au}$ from the star would still be only $\sim 1000 \mathrm{~K}$, cool enough for most dust species to exist at this location or closer in. It appears that its spectrum is steeper than Rayleigh-Jeans between 2 and $10 \mu \mathrm{m}$ (or its emission is variable). Such spectra can be generated by very small grains of carbon or of some of the robust oxides such as $\mathrm{FeO}$ expected to be produced in the destruction of silicate grains (Rieke et al. 2016; Kirchschlager et al. 2017). Another perspective on the HOSTS result is provided by the measurements with Spitzer and Herschel as analyzed by $\mathrm{Su}$ et al. (2013). They find that the debris SED indicates an asteroid-analog belt centered at $\sim 14$ au from the star. The LBTI measurement shows that the region interior to this belt must be largely devoid of dust, strengthening earlier mIR spectroscopic results ( $\mathrm{Su}$ et al. 2013). Given the strong dust emission in the nIR very close to the star, and in the mIR to fIR farther away, the void of dust at a few astronomical units from the star is particularly curious, and a clearing mechanism such as the presence of a planet might be required to explain it.

\section{Discussion}

In this section, we present a statistical analysis of the observations presented in this work. In Section 6.1 we derive and analyze basic detection statistics. We then constrain in Section 6.2 the median zodi levels for relevant groups of stars. In Section 6.3 we combine our observations with previous work to illustrate how detailed future modeling can constrain the exozodi luminosity function.

\subsection{Detection Rates among Different Subsamples}

To derive statistics from our observations, we first define relevant subsamples of our target stars. We exclude $\eta \mathrm{Crv}$ and $\beta$ Leo from the statistical analysis. While being part of the unbiased HOSTS target list, they were selected as commissioning targets specifically for their known $\mathrm{N}$-band excesses. They thus cannot be considered part of a statistically unbiased target selection before the majority of the HOSTS targets have been observed. $\beta \mathrm{UMa}$, despite its previous KIN detection, went through our real-time target selection during the observations and can be considered an unbiased target. Our sample can be
Table 3

Subsamples, Excess Detections, and Occurrence Rates

\begin{tabular}{lccc}
\hline \hline & Cold Dust & Clean & All \\
\hline \multirow{2}{*}{ Early type } & 1 of 3 & 1 of 9 & 2 of 12 \\
& $33_{-15}^{+28} \%$ & $11_{-4}^{+18} \%$ & $17_{-6}^{+15} \%$ \\
Sun-like & 2 of 2 & 1 of 14 & 3 of 16 \\
& $100_{-46}^{+0} \%$ & $7_{-2}^{+13} \%$ & $19_{-6}^{+13} \%$ \\
All & 3 of 5 & 2 of 23 & 5 of 28 \\
& $60_{-21}^{+16} \%$ & $8_{-3}^{+10} \%$ & $18_{-5}^{+9} \%$ \\
\hline
\end{tabular}

Table 4

Probability That Two Samples Are Drawn from the Same Distribution

\begin{tabular}{lcc}
\hline \hline Samples 1 & Sample 2 & Probability \\
\hline All early type & All Sun-like & 0.38 \\
All dusty & All clean & $0.03^{\mathrm{a}}$ \\
Clean early type & Dusty early type & 0.41 \\
Clean Sun-like & Dusty Sun-like & $0.03^{\mathrm{a}}$ \\
Clean early type & Clean Sun-like & 0.50 \\
Dusty early type & Dusty Sun-like & 0.30 \\
\hline
\end{tabular}

Note.

a These probabilities are significantly affected when considering $110 \mathrm{Her}$ a clean star (Section 5.2). In this case, the probability changes to 0.12 for "All dusty" versus "All clean" and to 0.19 for "Clean Sun-like" versus "Dusty Sun-like."

divided relatively evenly into early-type stars (spectral types F5 and earlier, 12 stars) and Sun-like stars (spectral types F6 and later, 16 stars). In addition, Mennesson et al. (2014) found from their KIN results that the detection rate of warm dust is higher for stars with previously known cold dust than for stars without. While with $\delta \mathrm{UMa}$ and $\theta$ Boo we find the first two cases of $\mathrm{mIR}$ interferometric excesses without previously known dust, our detection rates seem generally consistent with this conclusion. They also find a tentative anticorrelation between nIR-detected hot dust and their KIN detections. Because this was based on very small number statistics and our relevant sample is similarly small, we ignore the presence of hot dust for now and will discuss the hot dust systems separately at the end of this section. We thus divide our sample into early-type and late-type stars and into stars with previously known cold dust ("cold dust stars") and without ("clean stars"). Our derived occurrence rates of HZ dust at the sensitivity of the observations presented in this paper, and their binomial uncertainties, are listed in Table 3. To test whether the differences in detection rates measured from different subsamples are statistically significant, we perform Fisher's exact test (Table 4).

Most of our detection rates are consistent with each other given the statistical uncertainties from the limited sample sizes. However, we can rule out with a formally high confidence (probability 3\%) that the occurrence rate of $\mathrm{HZ}$ dust is the same among stars with and without cold dust, confirming the result by Mennesson et al. (2014). The result remains the same for Sun-like stars only, but no constraints can be put on early-type stars alone ( $41 \%$ probability).

Also interesting is the comparable detection rate for Sun-like and early-type stars, independent of the presence of cold dust. For Sun-like stars, our sensitivity in terms of zodi level is on average about four times worse than for early-type stars. Thus, a similar detection rate for Sun-like and early-type stars 
suggests a higher average dust level for Sun-like stars (but note the cautions in the following paragraph). If confirmed, this might imply that the transport of material to or through the HZ from farther out in the system is more efficient for Sun-like stars than for early-type stars. In particular, this could mean that Sun-like stars might harbor significant amounts of HZ dust even if no detectable amounts of cold dust are present, a conclusion also suggested by our detection on $\theta$ Boo (although our $\delta \mathrm{UMa}$ detection suggests that similar cases may exist for early-type stars as well). Such a scenario would complicate the target selection for future exo-Earth imaging missions.

It is important to note that our results are so far based on few detections mostly in the 3-5 $\sigma$ range and are affected by small number statistics. They thus require confirmation from a larger sample and more sensitive observations. In addition, the difference in detection rates between dusty and clean stars relies also on our ability to identify cold dust detections. For example, considering the detection of cold dust around $110 \mathrm{Her}$ as spurious due to background contamination and an underestimation of the measurement uncertainties would move this star with an LBTI detection to the clean stars sample. In this case, there is no significant difference in detection rates between clean and dusty stars with a probability of 0.12 that the two samples are drawn from the same occurrence rate. Furthermore, the detections around $\delta \mathrm{UMa}$ and $\theta$ Boo (both clean stars) demonstrate that limiting the target list of exo-Earth imaging surveys to stars without cold dust does not guarantee that all targets have low HZ dust levels, although we still find a lower detection rate around clean stars than around dusty ones.

Given the apparent correlation of $\mathrm{HZ}$ dust and cold dust, we need to exclude cold dust stars when searching for a correlation with the presence of hot dust. This limits our available sample of hot dust systems to only three stars, none of which shows any sign of excess related to emission close to the star in our observations. However, the small sample size prevents any conclusion on the correlation between hot dust and $\mathrm{HZ}$ dust. The discussion on Vega in Section 5.2 presents our strongest constraints that can be put on the hot dust systems from the available LBTI data without detailed modeling.

\subsection{Median Zodi Level}

The main goal of the HOSTS survey is to determine the typical HZ dust levels around nearby stars. Here, we perform a statistical analysis of the HOSTS targets observed so far in order to provide the strongest constraints possible at the moment. We follow the approach presented by Mennesson et al. (2014) to fit a probability distribution of the zodi levels (exozodi luminosity function) for our observed stars to our measurements using a maximum likelihood estimate. We assume a lognormal distribution for a given star to have a specific zodi level $z$ :

$$
p(z)=\frac{1}{z \varsigma \sqrt{2 \pi}} \exp \left(-\frac{(\ln z-\mu)^{2}}{2 \varsigma^{2}}\right) .
$$

The likelihood of finding the measured zodi levels for a sample of stars-given the assumed luminosity function and the individual uncertainties on each star-is computed for an equally spaced grid of values for the lognormal parameters $\mu$ and sigma parameter $\varsigma$. To derive the probability for the median of the fitted distribution, $m=\exp (\mu)$, and $\varsigma$, we then extend the approach used by Mennesson et al. (2014) by performing a
Bayesian analysis. We apply a $1 / m$ prior, equivalent to assuming a flat prior in $\mu$, marginalize the likelihood distribution over $\varsigma$, and compute the posterior cumulative probability distribution function (CPDF) of $m$. From this we can directly derive constraints on the median zodi level of our best-fit distribution for a sample of stars at any confidence level.

The alternative and more naive approach to derive a median of our measurements directly, rather than from a fit of the underlying distribution, does not yield good statistical results. For such an analysis one would need measurements, rather than upper limits, for all stars considered. This would force us to ignore our nondetections, which are most constraining for the underlying distribution, and to use only our few detections, which represent only the most extreme cases of the probability distribution. The use of a lognormal distribution is motivated by the fact that it has a well-defined median and a small number of parameters. We also tested the other distributions used by Mennesson et al. (2014): a uniform distribution with an upper cut-off value and a truncated Gaussian distribution. Both produce median zodi levels that are generally consistent with those from the lognormal distribution, while the uniform distribution does not reproduce our data well. In Section 6.3, we combine our results with available photometric constraints and compare them to a physical model of a luminosity function, which predicts a power-law distribution (Kennedy \& Wyatt 2013).

We perform this analysis on the zodi measurements obtained with the 13 pix and conservative apertures. The conservative apertures are used in addition to the formally more sensitive 13 pix aperture in order to test if potentially neglecting a fraction of the flux using the 13 pix aperture has any effect on our results. We still use the SZ model to convert null levels and uncertainties to zodi levels. We do not list the results in Table 2 because this is only a sanity check, and the zodi levels derived from this are not to be considered our final results. We will show below that the results from the 13 pix and conservative apertures are fully consistent.

As discussed before, we find a higher detection rate for stars with cold dust than for stars without. Although this does not preclude that the inner regions of some cold dust systems may be dust free, it disqualifies their host stars as good targets for an exo-Earth imaging survey. We thus concentrate our analysis on the clean stars in our sample, meaning stars without cold dust. ${ }^{16}$ We reach our best sensitivity in terms of zodi levels for earlytype stars, which will dominate our statistics. On the other hand, Sun-like stars are preferred targets for future exo-Earth imaging missions, because they are more numerous in the solar neighborhood and stellar suppression requirements become less stringent for detecting Earth-like planets orbiting them than early-type stars. It is unclear if the results for early-type stars can be applied to Sun-like stars, and the similar detection rates for both samples (despite lower sensitivity for Sun-like stars; see Section 6.1) suggest they might not. Thus, we perform the statistical analysis of the median zodi level for both the full sample of clean stars and for clean Sun-like stars only.

Maps of the likelihood derived for the searched parameter space of $m=\exp (\mu)$ and $\varsigma$ of the lognormal luminosity

\footnotetext{
${ }^{16}$ We ignore here the presence or absence of hot dust. The origin of this dust is still unclear, and we find no correlation between the presence of hot dust and our detections.
} 

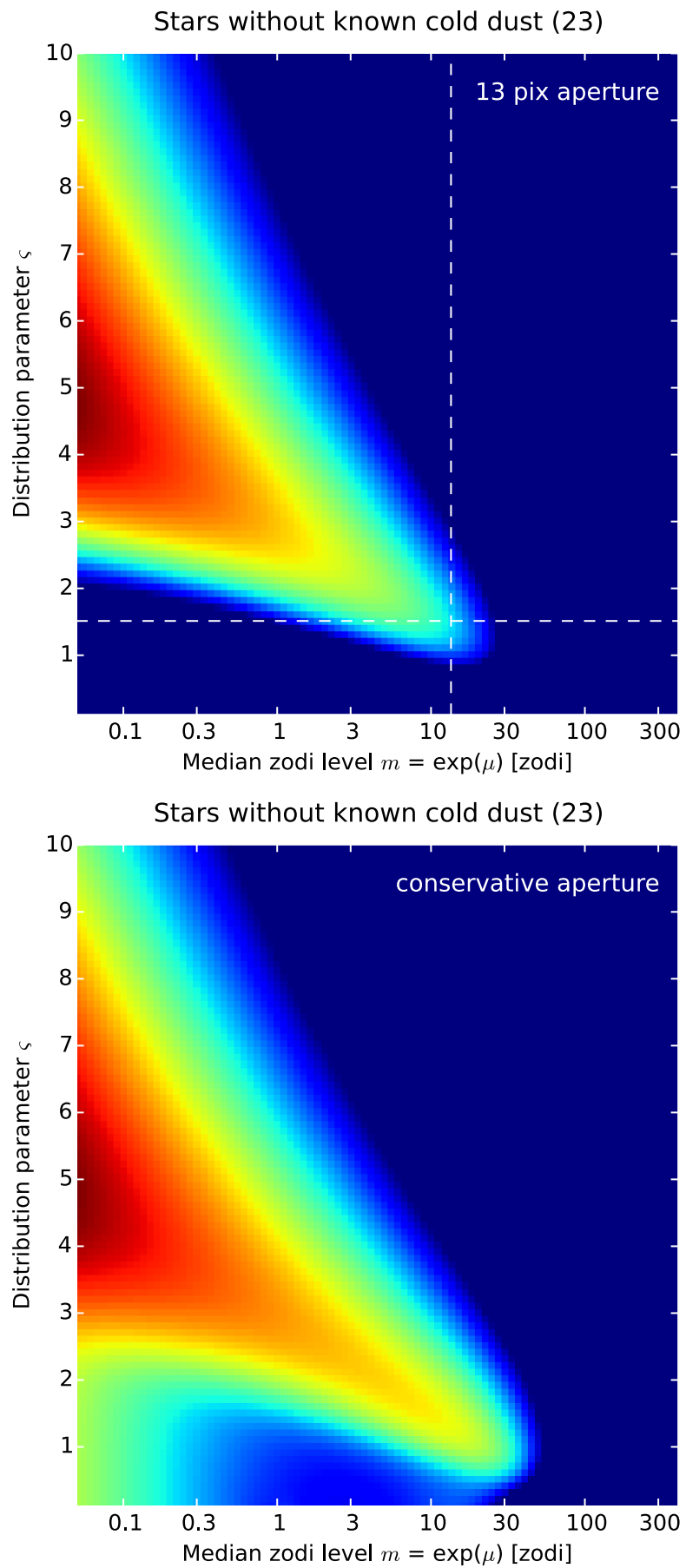

Sun-like stars without known cold dust (14)

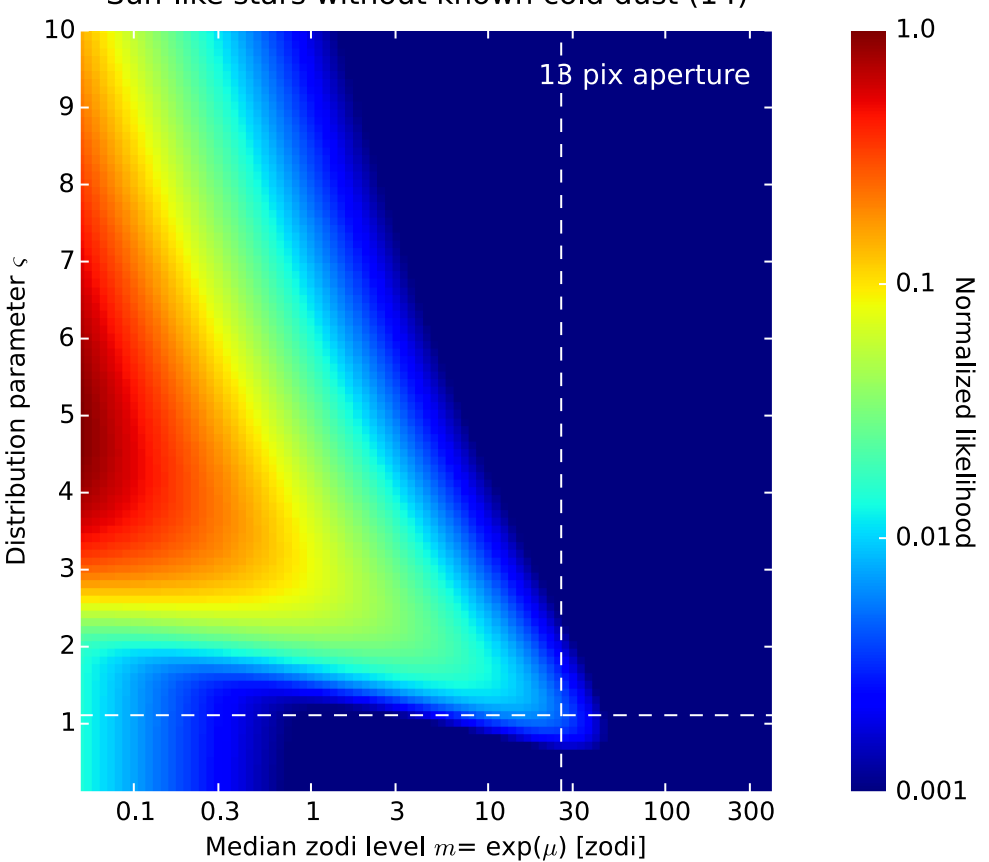

Sun-like stars without known cold dust (14)

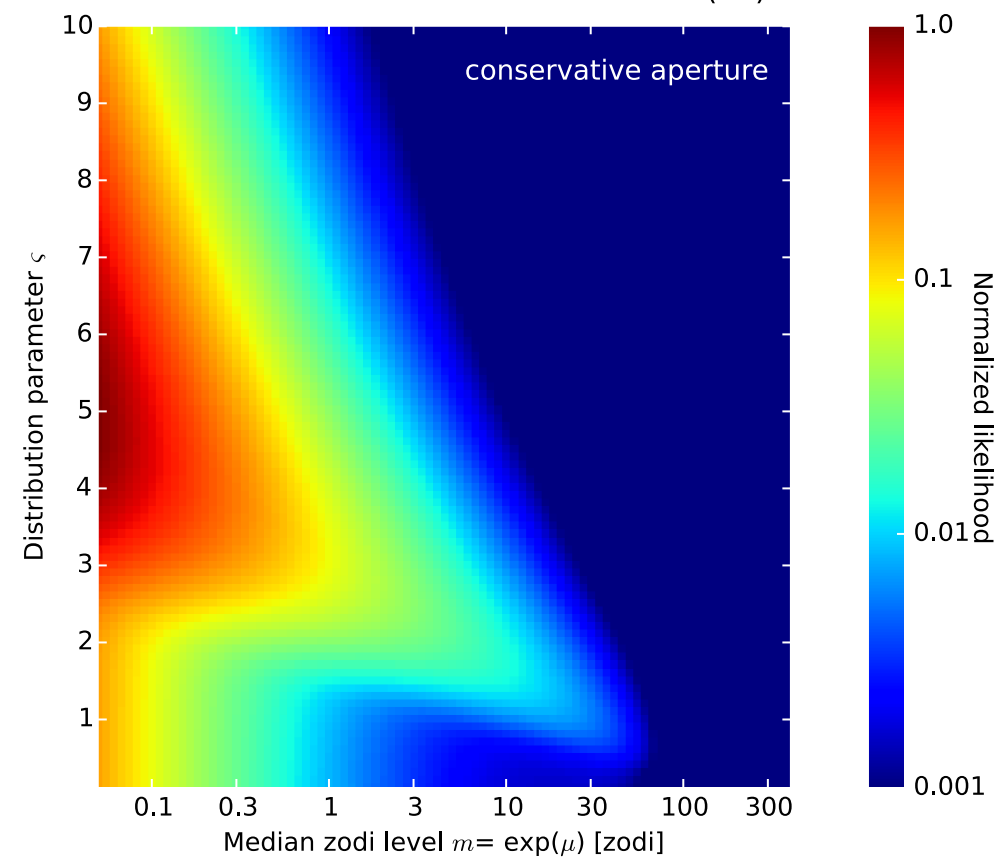

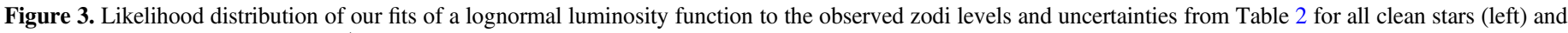

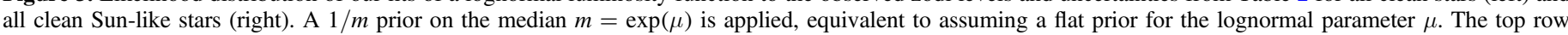

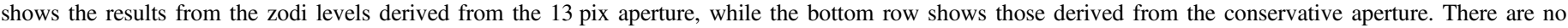

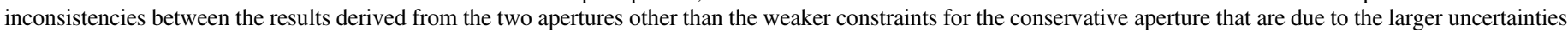

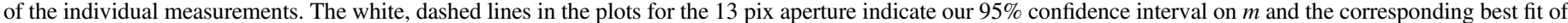

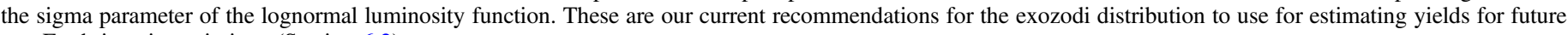
exo-Earth imaging missions (Section 6.2).

function with the $1 / m$ prior applied are shown in Figure 3 for both samples and for the 13 pix and conservative apertures. Very small values of $\varsigma$ are unable to fit the data well because our detections of excesses around $\delta \mathrm{UMa}$ and $\theta$ Boo show that there can still be a significant amount of warm dust present even for stars without detectable mIR to fIR excess. Thus, a narrow probability distribution described by a small $\varsigma$ is not able to reproduce the data. A large median is inconsistent with the large number of nondetections. For intermediate values of $\varsigma$ and $m$, a larger value of $\varsigma$ in combination with smaller $m$ and vice versa provides fits of similar quality to the data, because both cases are able to produce a range of excess significances given our sensitivity. We find no inconsistencies between the results for the 13 pix and conservative apertures, while the constraints from the latter are weaker due to the larger uncertainties on the individual measurements. 
The overall shape of our likelihood distribution, and in particular the "nose" of relatively high likelihood around $\varsigma=2$ and $m=10$, is dominated by the detections around $\delta \mathrm{UMa}$ and $\theta$ Boo. The detection around 110 Her would be another such case if the unclear detection of faint cold dust was considered spurious. Even for stars without known cold dust, we now start to measure the underlying luminosity function. Thus, further increasing the sensitivity will result in a better measurement of the luminosity function rather than improved upper limits. The shape of the likelihood distribution also shows a degeneracy in our fits to the data and suggests that a lognormal distribution might not be a good approximation of the actual luminosity function. We find more evidence for this when comparing the results from the Bayesian analysis with those from a pure maximum-likelihood estimate as performed by Mennesson et al. (2014). While the Bayesian analysis suggests that a zero median is the best fit to the data, the likelihood peaks at $m=7_{-6}^{+8}$ zodis for the full sample of clean stars and $m=13_{-12}^{+23}$ zodis for clean, Sun-like stars. If there were no degeneracies and the lognormal distribution was a good fit to the data, the results from the two approaches should be the same. We note, however, that the difference between the two methods is only at the $\sim 1 \sigma$ level and that the two detections around $\delta \mathrm{UMa}$ and $\theta$ Boo at the $\sim 4 \sigma$ level do not allow for any strong conclusions on the actual luminosity function.

A viable alternative to a broad, lognormal luminosity function or similar single-peaked or monotonous distributions would be a bimodal one, where the majority of systems have relatively low zodi levels but a small number of stars are surrounded by a significant amount of warm dust. Stars without detections of cold dust but with detected HZ dust might, for example, harbor a Kuiper Belt or asteroid belt analog that is massive enough to sustain a high zodi level through inward migration of dust grains, but that is too faint to be detected in available data. Stars without large amounts of $\mathrm{HZ}$ dust might not have a cold dust belt at all or might harbor one or more giant planets between the cold belt and the $\mathrm{HZ}$ that prevent dust from migrating inward in large amounts. Confirming a bimodal luminosity function (e.g., in the light of potentially higher dust levels around Sun-like stars compared to early-type stars) and identifying stars belonging to the "high zodi level" and "low zodi level" categories would thus be most valuable for our understanding of the architectures and dynamics of planetary systems. Moreover, it would be favorable for exo-Earth imaging surveys, because (1) the majority of the targets would have a relatively low zodi level and (2) the stars surrounded by a large amount of dust could be identified prior to the mission by extensive target vetting with the LBTI and similar instruments (e.g., the hi-5 concept at the Very Large Telescope Interferometer, Defrère et al. 2018) in the next two decades.

We illustrate these advantages by excluding $\delta \mathrm{UMa}$ and $\theta$ Boo from our samples and repeating the median zodi analysis. We do this for early-type and Sun-like stars separately. The resulting likelihood maps are shown in Figure 4 . The nose toward large $m$ and small $\varsigma$ disappears for both samples. The upper limits on the median zodi levels on these samples thus improve by a factor of about two. Thus, our upper limits for stars identified to belong to the "low zodi level" category are approximately twice as strong as for stars not vetted by LBTI observations. Discriminating between these two scenarios requires a larger sample and more sensitive observations. Both can be provided by extending the HOSTS survey (larger sample and better sensitivity due to completed observations and higher data quality from the set-point modulation and more experience with the data acquisition compared to some of the data presented in this work). Specifically, we estimate that completing the observations for all stars in this paper and observing an equal number of additional stars will suffice to conclude if stars like $\delta \mathrm{UMa}$ and $\theta$ Boo are outliers or can be explained by the high excess tail of a lognormal or similar distribution.

From the available data, we derive upper limits on the median zodi levels in our samples using the results from our Bayesian analysis. The CPDFs derived for the samples of all clean stars and clean Sun-like stars using the 13 pix aperture are shown in Figure 5. For all clean stars, we find upper limits of $m=13$ zodis and $m=21$ zodis at $95 \%$ and $99 \%$ confidence. For clean Sun-like stars, we find upper limits of $m=26$ zodis and $m=47$ zodis, respectively. From our results, we suggest the use of conservative but not overly pessimistic assumptions on the exozodi luminosity function when simulating yields for future exo-Earth imaging surveys. A lognormal distribution with $m$ equivalent to our $95 \%$ confidence level and the corresponding best-fit value of $\varsigma$ seem appropriate. For all clean stars, these parameters are $m=13$ zodis and $\varsigma=1.5$. For clean Sun-like stars, the parameters are $m=26$ zodis and $\varsigma=1.2$.

\subsection{Constraints on the Exozodi Luminosity Function}

In the previous section, we put a constraint on the median zodi level of different samples of stars from our observations. For this, we assumed a lognormal distribution. Strong constraints on the actual shape of the luminosity function are not possible based on our limited sample and number of detections. However, our results constrain the faintest currently reachable regime of the luminosity function and can be combined with available constraints on the bright end. The cleanest such statistics focusing specifically on HZ dust have been derived from a sample of Wide-field Infrared Survey Explorer (WISE) observations by Kennedy \& Wyatt (2013). We compare the sensitivity to zodi levels and the sample size of the LBTI, the WISE sample, and the KIN data in Figure 6.

The observable used by Kennedy \& Wyatt (2013) for the statistical analysis and the presentation of the modeling results is the disk-to-star flux ratio, rather than the zodi level. We thus first convert our LBTI zodi levels to disk-to-star flux ratios. We use again our SZ model, but note that this conversion is more uncertain than deriving the zodi level from the null measurements, because we now extrapolate from the spatially filtered LBTI excess measurements to photometric excesses, which are more sensitive to the spatial dust distribution (radial slope, inner edge). Converting WISE excesses to zodi levels as was done to create Figure 6 is equally affected by the same uncertainties. Moreover, this would impact already published data, require detailed information on the much larger WISE sample, and complicate the comparison to the model presented by Kennedy \& Wyatt (2013). We then plot the occurrence rate of exozodiacal dust inferred from our observations for all stars and all Sun-like stars (removing $\eta \mathrm{Crv}$ and $\beta$ Leo as described in Section 6.1) over the disk-to-star flux ratio together with the detection rates from Kennedy \& Wyatt (2013). The result is shown in Figure 7. The conversion from zodi level to flux ratio eliminates the sensitivity advantage to HZ dust of the LBTI for early-type stars. 

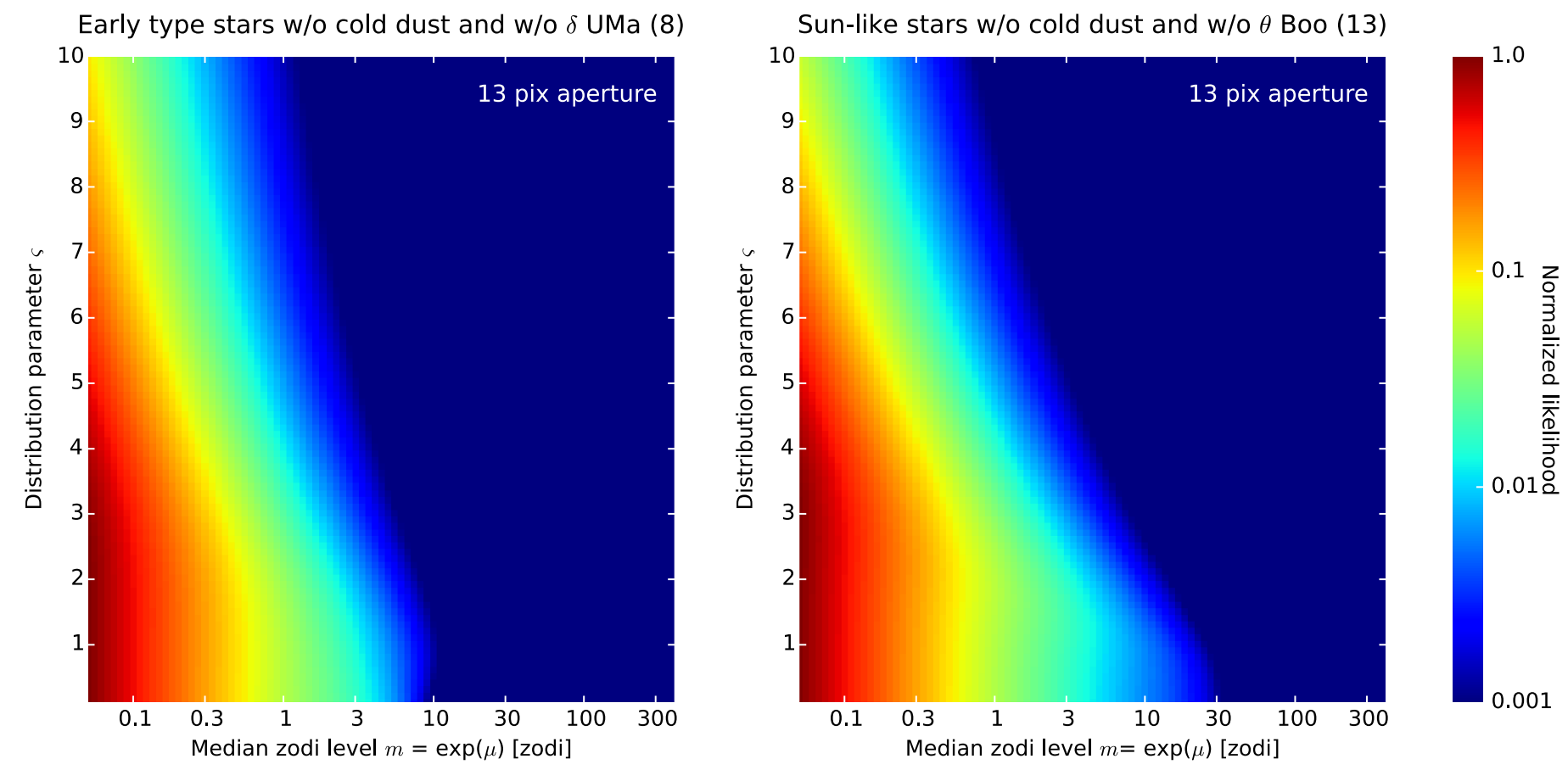

Figure 4. Same as Figure 3, but for the samples of clean early-type stars excluding $\delta$ UMa (left) and clean Sun-like stars excluding $\theta$ Boo (right).

We also plot the two-component in situ model of an initial dust belt evolving over time and of random, additional dust production over the star's lifetime. This model predicts a power-law slope of the luminosity function with an exponent of -1 . We find that our inferred occurrence rate is higher than predicted by the model; a power-law slope of -2 seems better suited to reproduce the data. This might suggest that an additional dust-delivery mechanism is at play for low dust levels in the range of a few zodis to several tens of zodis. This could, for example, be explained by dust delivery through cometary activity or from an outer dust belt through PoyntingRobertson and stellar wind drag that can only sustain dust levels up to a maximum surface density for a given system configuration (e.g., Kennedy \& Piette 2015). A detailed analysis of the samples and evolutionary modeling is necessary for a better understanding of this behavior of the luminosity function but is beyond the scope of this paper.

It is important to note that an extrapolation from the WISE and LBTI rates to the occurrence rate at lower zodi levels cannot be compared to our median zodi analysis in the previous section. For the median zodi analysis, we focused on stars without known cold dust, which are thought to be good targets for exo-Earth imaging, while here we consider all stars, for which we find a higher detection rate in Section 6.1.

\section{Conclusions}

We have presented the first statistical results from the HOSTS survey for HZ dust around nearby stars. Our sensitivity for individual, completed targets is a factor of 5-10 better than previous observations. Although only a limited sample of stars have been observed so far, the statistical constraints from our survey are already two to five times stronger than earlier results.

We find four new detections, resulting in an overall detection rate of $18 \%$. This means we are now reaching a sensitivity at which statistical samples of stars with HZ dust can be created, similar to fIR observations of debris disks and nIR observations of hot exozodiacal dust. We find the first three detections around Sun-like stars and the first two around stars without any previously known circumstellar dust. Our inferred occurrence rate at LBTI's sensitivity is $18_{-5}^{+9} \%$ for all stars and similar for early-type stars and Sun-like stars $\left(17_{-6}^{+15} \%\right.$ versus $\left.19_{-6}^{+13} \%\right)$. It is significantly different for stars with and without a previously known Kuiper Belt-like disk of cold dust $\left(60_{-21}^{+16} \%\right.$ versus $8_{-3}^{+10} \%$ ), confirming earlier results at higher sensitivity. Interestingly, the similar detection rate around early-type and Sun-like stars comes at a four times lower sensitivity to HZ dust around Sun-like stars, which might suggest that the HZs of Sun-like stars are in general dustier than those of early-type stars. This tentative result, however, is derived from a small number of detections mostly at the $3-5 \sigma$ level. It thus needs confirmation from a larger sample and more sensitive observations. A detailed analysis of the detected systems might also reveal an alternative explanation.

A most puzzling result is our nondetection of warm dust around Vega, for which massive asteroid belt and Kuiper Belt analogs have been detected in the mIR to fIR and a large amount of hot dust has been detected in the nIR. This raises the question of what mechanism clears the region between $\sim 0.5$ au and $\sim 5$ au from the star of dust.

A statistical analysis of our sample-assuming a lognormal luminosity function-puts upper limits on the median zodi level of stars without previously known cold dust to 13 zodis and 21 zodis at $95 \%$ and $99 \%$ confidence, respectively. For Sun-like stars only, the corresponding limits are 26 zodis and 47 zodis. We demonstrate that these limits are no longer governed by the measurement uncertainties, as was the case for earlier work, but by the discovery of a few systems with detected excesses despite the absence of detectable amounts of cold dust. We note the possibility that the actual underlying 

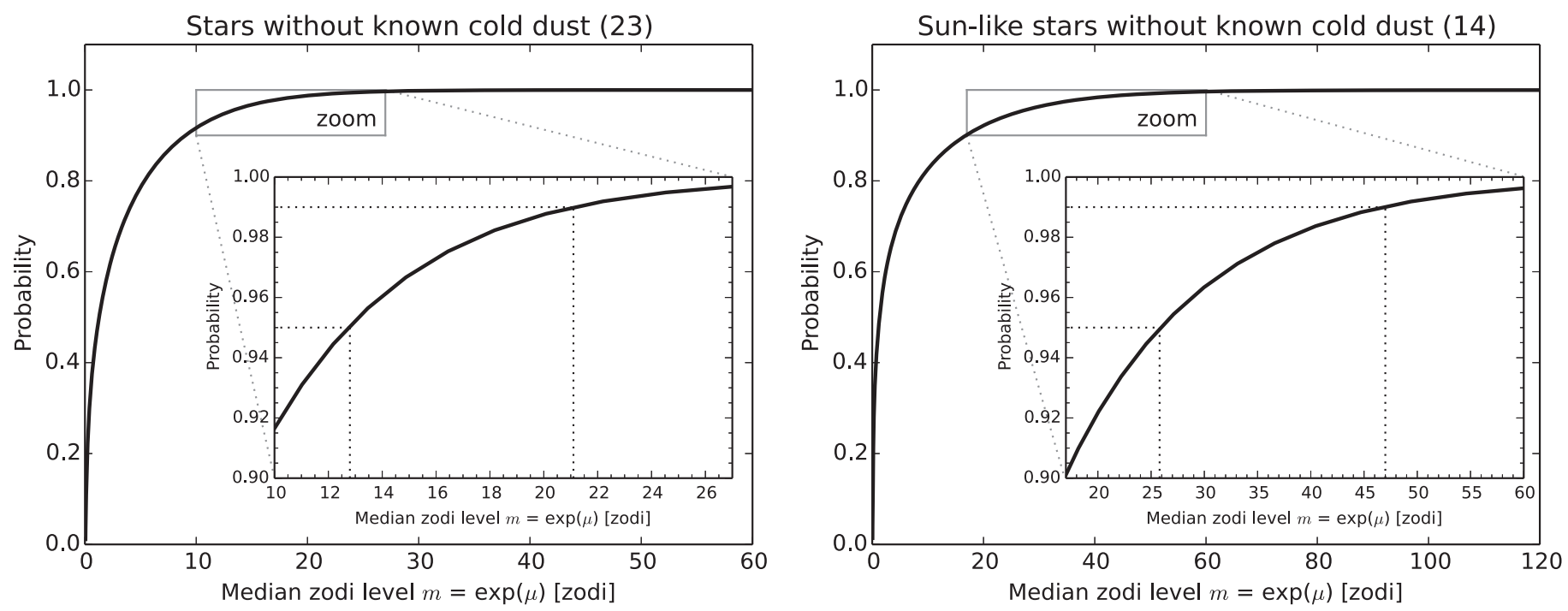

Figure 5. CPDFs of the median zodi levels from Table 2 for our sample of all clean stars (left) and clean Sun-like stars (right). The full CPDFs are shown in the large plot, and a zoom into the relevant region to determine the $95 \%$ and $99 \%$ confidence intervals is shown in the inlay in each plot. Dotted horizontal and vertical lines mark the $95 \%$ and $99 \%$ confidence levels and corresponding median zodi levels.

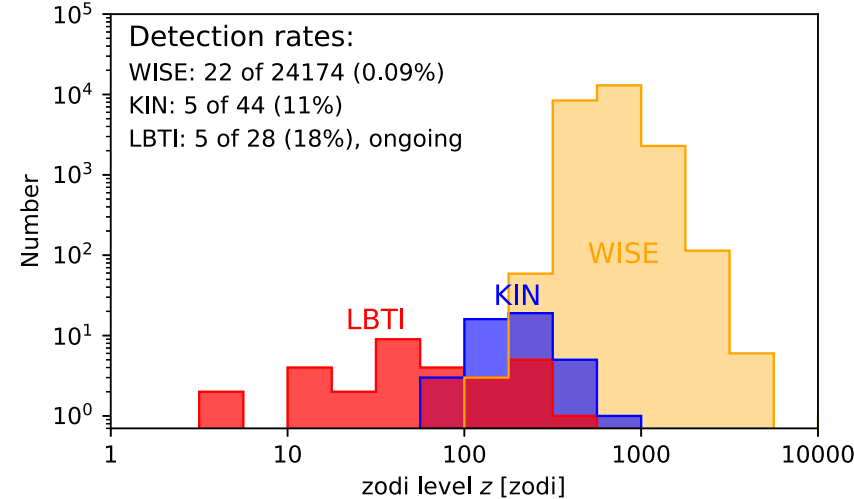

Figure 6. Distribution of sensitivity to HZ dust and sample sizes of LBTI, KIN, and the WISE sample from Kennedy \& Wyatt (2013). Combining these samples will allow for a comprehensive analysis of the exozodi luminosity function over the range of a few zodis to its brightest specimen at several thousand zodis.

distribution might be bimodal, rather than lognormal, including a few systems with large amounts of HZ dust and the majority of systems with little such dust. We estimate the limit that can be put on the median zodi level of a target list for an exo-Earth imaging survey that has been fully vetted by LBTI or similar observations. We find that it would already be approximately twice as strong as for stars without LBTI observations and will further improve with more stars being observed. Thus, constraining the exozodi luminosity function by increasing the available sample size and improving the sensitivity of the observations is critical. Both can be achieved with an extended HOSTS survey, and extensive vetting of future exo-Earth imaging targets can be done with the LBTI and similar facilities.

Comparing our inferred occurrence rates of $\mathrm{HZ}$ dust in the faint regime of the luminosity function with previous photometric results for the bright end suggests that its slope is steeper than predicted by a model of in situ dust production. This suggests an additional dust-delivery mechanism at lower dust levels than could be detected photometrically.

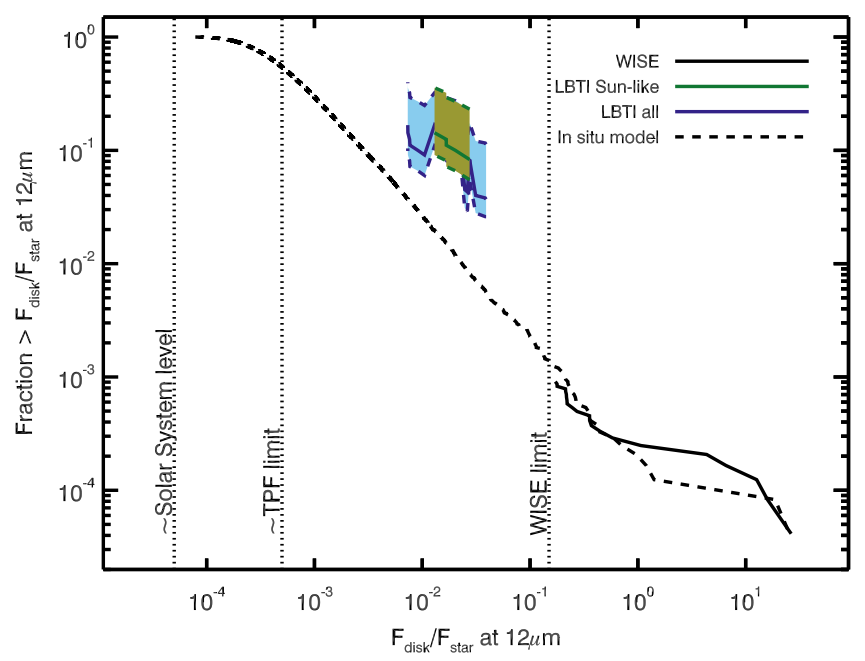

Figure 7. Exozodi luminosity function constrained by our LBTI statistics and the WISE sample by Kennedy \& Wyatt (2013). Occurrence rates and sensitivities for Sun-like and all stars are consistent, so the two curves lie on top of each other. The slope from the WISE detection rate to the one from the LBTI appears steeper than the prediction from the in situ dust production model by Kennedy \& Wyatt (2013).

From our current results, we suggest using a lognormal exozodi luminosity function with conservative but not overly pessimistic parameters when simulating yields for future exoEarth imaging missions. A combination of our $95 \%$ confidence upper limit on the median $m$ and the corresponding best-fit value of the sigma parameter $\varsigma$ of the distribution seems appropriate. From our results using all stars without known cold dust, these parameters are $m=13$ zodi and $\varsigma=1.5$. For Sun-like stars only, the parameters are $m=26$ zodis and $\varsigma=1.2$.

The Large Binocular Telescope Interferometer is funded by the National Aeronautics and Space Administration as part of its Exoplanet Exploration Program. The LBT is an international collaboration among institutions in the United States, Italy, and Germany. LBT Corporation partners are The University of 
Arizona on behalf of the Arizona university system; Instituto Nazionale di Astrofisica, Italy; LBT Beteiligungsgesellschaft, Germany, representing the Max-Planck Society, the Astrophysical Institute Potsdam, and Heidelberg University; The Ohio State University; and The Research Corporation, on behalf of The University of Notre Dame, University of Minnesota and University of Virginia. This research has made extensive use of the SIMBAD database (Wenger et al. 2000) and the VizieR catalog access tool (Ochsenbein et al. 2000), both operated at CDS, Strasbourg, France; of Python, including the NumPy, SciPy, Matplotlib (Hunter 2007); and Astorpy (Astropy Collaboration et al. 2013) libraries; and of NASA's Astrophysics Data System Bibliographic Services. G.M.K. is supported by the Royal Society as a Royal Society University Research Fellow. A.S. is partially supported by funding from the Center for Exoplanets and Habitable Worlds. The Center for Exoplanets and Habitable Worlds is supported by the
Pennsylvania State University, the Eberly College of Science, and the Pennsylvania Space Grant Consortium.

Facility: LBT:LBTI.

\section{Appendix}

In addition to the description of the observations in Section 3 and the null measurements, the observing dates of our targets, calibrators used, hour angle range covered, and data quality provide important context for our measurements. For easy access and future reference, we provide in Table 5 a brief observing log containing this information. All raw and calibrated HOSTS data will be available to the public one year after the observation date through the LBTI Archive (http://lbti.ipac.caltech.edu/), from where more detailed information can be extracted.

Table 5

Observing Log

\begin{tabular}{|c|c|c|c|c|c|}
\hline HD \# & Name & UT Date & Calibrators HD \# & HA Range (h) & Comments \\
\hline \multirow[t]{2}{*}{10476} & 107 Psc & 2016 Nov 14 & 7087 & $-0.24 \ldots 0.59$ & average data quality \\
\hline & & 2016 Nov 16 & 7318,6953 & $\begin{array}{llll}-0.71 \ldots & 0.34\end{array}$ & mediocre data quality \\
\hline 16160 & GJ 105 A & 2016 Nov 15 & 21051,13596 & $0.38 \ldots 1.04$ & mediocre data quality \\
\hline \multirow[t]{2}{*}{22049} & $\epsilon$ Eri & 2014 Nov 09 & 18322,29065 & $2.06 \ldots 2.59$ & com., low data quality \\
\hline & & 2014 Nov 10 & 18322,23249 & $0.29 \ldots 1.07$ & com., mediocre data quality \\
\hline 30652 & 1 Ori & 2017 Feb 09 & 31421,31767 & $\begin{array}{llll}-0.03 & \ldots & 0.99\end{array}$ & bkg., low data quality \\
\hline 33111 & $\beta$ Eri & 2017 Feb 10 & 31767,36780 & $1.08 \ldots 2.02$ & average data quality \\
\hline 34411 & $\lambda$ Aur & 2017 Jan 29 & 38656,40441 & $1.55 \ldots 3.26$ & high data quality \\
\hline \multirow[t]{2}{*}{48373} & $\xi \mathrm{Gem}$ & 2016 Nov 14 & 49968,48433 & $-0.09 \ldots 0.64$ & average data quality \\
\hline & & 2016 Nov 15 & 52960 & $-1.63 \ldots 0.70$ & mediocre data quality \\
\hline \multirow[t]{2}{*}{81937} & $23 \mathrm{UMa}$ & 2016 Nov 15 & 86378 & $\begin{array}{llll}-0.88 & \ldots & 0.17\end{array}$ & mediocre data quality \\
\hline & & 2017 Feb 11 & 73108,92424 & $-2.54 \ldots-1.64$ & good data quality \\
\hline 88230 & GJ 380 & 2017 Apr 06 & 86378,95212 & $-0.96 \ldots-0.27$ & good data quality \\
\hline 89449 & 40 Leo & 2017 Feb 09 & 89024,93257 & $-1.98 \ldots-0.36$ & bkg., low data quality \\
\hline 95418 & $\beta \mathrm{UMa}$ & 2017 Apr 03 & $86378,94247,95212$ & $-1.16 \ldots 0.98$ & good data quality \\
\hline \multirow[t]{2}{*}{97603} & $\delta$ Leo & 2017 Feb 10 & 99902,94336 & $-1.61 \ldots-0.99$ & good data quality \\
\hline & & 2017 May 12 & 99169,98262 & $0.54 \ldots 1.22$ & good data quality \\
\hline 102647 & $\beta$ Leo & 2015 Feb 08 & $104979,109742,108381$ & $1.33 \ldots 2.92$ & com., good data quality \\
\hline \multirow[t]{2}{*}{103287} & $\gamma \mathrm{UMa}$ & 2017 Apr 06 & 94247,95212 & $-0.47 \ldots-0.03$ & good data quality \\
\hline & & 2017 May 01 & 102224,107274 & $2.09 \ldots 2.76$ & good data quality \\
\hline \multirow[t]{2}{*}{106591} & $\delta \mathrm{UMa}$ & 2017 Feb 09 & 107465,102328 & $-0.78 \ldots 0.35$ & average data quality \\
\hline & & 2017 May 21 & 101673,113092 & $0.60 \ldots 1.60$ & mediocre data quality \\
\hline 108767 & $\delta \mathrm{Crv}$ & 2017 Feb 10 & 114113,111500 & $-1.19 \ldots-0.52$ & mediocre data quality \\
\hline 109085 & $\eta \mathrm{Crv}$ & 2014 Feb 12 & $108522,107418,109272$ & $-0.28 \ldots 2.18$ & com., low data quality \\
\hline 120136 & $\tau$ Boo & 2017 May 12 & 114326,125560 & $0.62 \ldots 2.19$ & average data quality \\
\hline \multirow[t]{2}{*}{126660} & $\theta$ Boo & 2017 Feb 09 & 128902 & $-0.29 \ldots 0.35$ & bkg., low data quality \\
\hline & & 2017 Apr 11 & 128902,138265 & $-0.56 \ldots 0.76$ & good data quality \\
\hline \multirow[t]{2}{*}{128167} & $\sigma$ Boo & 2017 Apr 03 & 133392 & $-0.39 \ldots 0.68$ & low data quality \\
\hline & & 2017 Apr 06 & 126597,129972 & $-1.22 \ldots-0.59$ & average data quality \\
\hline 129502 & $\mu$ Vir & 2017 Feb 10 & $131477,133165,130952$ & $-1.43 \ldots 0.16$ & average data quality \\
\hline 141004 & $\lambda$ Ser & 2017 May 01 & 145892,145085 & $0.24 \ldots 0.90$ & good data quality \\
\hline 142373 & $\chi$ Her & 2017 Apr 11 & $137704,144204,137704$ & $0.83 \ldots 2.29$ & good data quality \\
\hline \multirow[t]{2}{*}{142860} & $\gamma$ Ser & 2017 Apr 06 & 149009,142574 & $-0.85 \ldots-0.18$ & good data quality \\
\hline & & 2017 May 21 & 141992,145892 & $-0.50 \ldots 0.18$ & good data quality \\
\hline 172167 & $\alpha$ Lyr & 2017 Apr 06 & 164646,163770 & $-1.25 \ldots-0.61$ & good data quality \\
\hline 173667 & $110 \mathrm{Her}$ & 2017 Apr 08 & 170951,176527 & $-1.69 \ldots-0.54$ & average data quality \\
\hline 185144 & $\sigma$ Dra & 2017 May 01 & 191277,170693 & $-1.81 \ldots-0.84$ & good data quality \\
\hline 187642 & $\alpha \mathrm{Aql}$ & 2017 May 12 & $184406,189695,192107$ & $-2.65 \ldots 0.20$ & sat., bkg., mediocre data quality \\
\hline 203280 & $\alpha$ Cep & 2016 Oct 16 & 198149,209960 & $2.20 \ldots 3.11$ & mediocre data quality \\
\hline \multirow[t]{2}{*}{215648} & $\xi$ Peg A & 2016 Nov 14 & 218792 & $0.47 \ldots 1.74$ & mediocre data quality \\
\hline & & 2016 Nov 16 & 209167, 220009 & $-0.23 \ldots 0.73$ & mediocre data quality \\
\hline
\end{tabular}

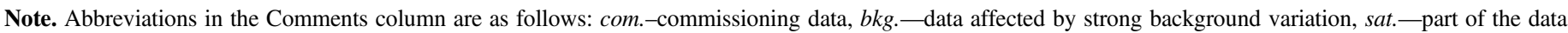
unusable due to saturation. In addition to these specific cases, the data quality is judged based on the uncertainty of the null measurements. 


\section{ORCID iDs}

S. Ertel (1D https://orcid.org/0000-0002-2314-7289

D. Defrère (i) https://orcid.org/0000-0003-3499-2506

B. Mennesson (iD https://orcid.org/0000-0003-4205-4800

G. M. Kennedy (i) https://orcid.org/0000-0001-6831-7547

G. Rieke (i) https://orcid.org/0000-0003-2303-6519

A. Shannon (iD https://orcid.org/0000-0002-0711-4516

E. Spalding (iD https://orcid.org/0000-0003-3819-0076

J. M. Stone (i) https://orcid.org/0000-0003-0454-3718

A. J. Weinberger (iD https://orcid.org/0000-0001-6654-7859

O. Absil (iD https://orcid.org/0000-0002-4006-6237

V. P. Bailey (iD https://orcid.org/0000-0002-5407-2806

A. Gaspar (i) https://orcid.org/0000-0001-8612-3236

J. M. Leisenring (1) https://orcid.org/0000-0002-0834-6140

R. Millan-Gabet (i) https://orcid.org/0000-0003-0447-5866

K. M. Morzinski (iD https://orcid.org/0000-0002-1384-0063

E. Pinna (1) https://orcid.org/0000-0002-6243-5697

A. Roberge (10) https://orcid.org/0000-0002-2989-3725

A. J. Skemer (iD https://orcid.org/0000-0001-6098-3924

K. Y. L. Su (i) https://orcid.org/0000-0002-3532-5580

M. C. Wyatt (iD https://orcid.org/0000-0001-9064-5598

\section{References}

Absil, O., Defrère, D., Coudé du Foresto, V., et al. 2013, A\&A, 555, A104 Absil, O., di Folco, E., Mérand, A., et al. 2006, A\&A, 452, 237

Akeson, R. L., Ciardi, D. R., Millan-Gabet, R., et al. 2009, ApJ, 691, 1896 Anglada-Escudé, G., \& Butler, R. P. 2012, ApJS, 200, 15

Astropy Collaboration, Robitaille, T. P., Tollerud, E. J., et al. 2013, A\&A, 558, A 33

Aufdenberg, J. P., Mérand, A., Coudé du Foresto, V., et al. 2006, ApJ, 645,664

Aumann, H. H. 1985, PASP, 97, 885

Aumann, H. H. 1988, AJ, 96, 1415

Aumann, H. H., Beichman, C. A., Gillett, F. C., et al. 1984, ApJL, 278, L23

Backman, D., Marengo, M., Stapelfeldt, K., et al. 2009, ApJ, 690, 1522

Backman, D. E., \& Paresce, F. 1993, in Protostars and Planets III, ed. E. H. Levy \& J. I. Lunine (Baltimore, MD: STScI), 1253

Bailey, V. P., Hinz, P. M., Puglisi, A. T., et al. 2014, Proc. SPIE, 9148, 914803 Barnes, S. A. 2007, ApJ, 669, 1167

Beichman, C. A., Bryden, G., Stapelfeldt, K. R., et al. 2006, ApJ, 652, 1674 Benedict, G. F., McArthur, B. E., Gatewood, G., et al. 2006, AJ, 132, 2206

Böhm, M., Pott, J.-U., Borelli, J., et al. 2016, Proc. SPIE, 9906, 99062R

Bonsor, A., Raymond, S. N., Augereau, J.-C., \& Ormel, C. W. 2014, MNRAS, 441, 2380

Booth, M., Dent, W. R. F., Jordán, A., et al. 2017, MNRAS, 469, 3200

Booth, M., Kennedy, G., Sibthorpe, B., et al. 2013, MNRAS, 428, 1263

Bordé, P., Coudé du Foresto, V., Chagnon, G., \& Perrin, G. 2002, A\&A, 393, 183

Brogi, M., Marzari, F., \& Paolicchi, P. 2009, A\&A, 499, L13

Chavez-Dagostino, M., Bertone, E., Cruz-Saenz de Miera, F., et al. 2016, MNRAS, 462, 2285

Chelli, A., Duvert, G., Bourgès, L., et al. 2016, A\&A, 589, A112

Chen, C. H., Sargent, B. A., Bohac, C., et al. 2006, ApJS, 166, 351

Churcher, L. J., Wyatt, M. C., Duchêne, G., et al. 2011, MNRAS, 417, 1715

Crooke, J. A., Roberge, A., Domagal-Goldman, S. D., et al. 2016, Proc. SPIE, 9904, 99044R

Danchi, W., Bailey, V., Bryden, G., et al. 2014, Proc. SPIE, 9146, 914607

Defrère, D., Absil, O., Augereau, J.-C., et al. 2011, A\&A, 534, A5

Defrère, D., Absil, O., Berger, J.-P., et al. 2018, arXiv:1801.04148

Defrère, D., Absil, O., den Hartog, R., Hanot, C., \& Stark, C. 2010, A\&A, 509, A9

Defrère, D., Hinz, P. M., Mennesson, B., et al. 2016, ApJ, 824, 66

Defrère, D., Hinz, P. M., Skemer, A. J., et al. 2015, ApJ, 799, 42

Defrère, D., Lebreton, J., Le Bouquin, J.-B., et al. 2012, A\&A, 546, L9

Dermott, S. F., Kehoe, T. J. J., Durda, D. D., Grogan, K., \& Nesvorný, D. 2002, in ESA Special Publication 500, Asteroids, Comets, and Meteors: ACM 2002, ed. B. Warmbein (Noordwijk: ESA), 319

Duchêne, G., Arriaga, P., Wyatt, M., et al. 2014, ApJ, 784, 148

Durkan, S., Janson, M., \& Carson, J. C. 2016, ApJ, 824, 58
Eiroa, C., Marshall, J. P., Mora, A., et al. 2011, A\&A, 536, L4

Eiroa, C., Marshall, J. P., Mora, A., et al. 2013, A\&A, 555, A11

Ertel, S., Absil, O., Defrère, D., et al. 2014, A\&A, 570, A128

Ertel, S., Defrère, D., Absil, O., et al. 2016, A\&A, 595, A44

Ertel, S., Wolf, S., \& Rodmann, J. 2012, A\&A, 544, A61

Faramaz, V., Ertel, S., Booth, M., Cuadra, J., \& Simmonds, C. 2017, MNRAS, 465,2352

Fischer, D. A., Marcy, G. W., \& Spronck, J. F. P. 2014, ApJS, 210, 5

Frolov, M. S. 1970, IBVS, 427, 1

Gáspár, A., \& Rieke, G. H. 2014, ApJ, 784, 33

Gáspár, A., Rieke, G. H., \& Balog, Z. 2013, ApJ, 768, 25

Gezari, D. Y., Schmitz, M., Pitts, P. S., \& Mead, J. M. 1993, Catalog of Infrared Observations (3rd ed.; Greenbelt, MD: NASA)

Gillett, F. C. 1986, in Light on Dark Matter, Vol. 124, ed. F. P. Israel (Dordrecht: Reidel), 61

Greaves, J. S., Holland, W. S., Moriarty-Schieven, G., et al. 1998, ApJL, 506, L133

Greaves, J. S., Sibthorpe, B., Acke, B., et al. 2014, ApJL, 791, L11

Gulliver, A. F., Hill, G., \& Adelman, S. J. 1994, ApJL, 429, L81

Hanot, C., Mennesson, B., Martin, S., et al. 2011, ApJ, 729, 110

Hatzes, A. P., Cochran, W. D., McArthur, B., et al. 2000, ApJL, 544, L145

Hinz, P. M., Defrère, D., Skemer, A., et al. 2016, Proc. SPIE, 9907, 990704

Hoffmann, W. F., Hinz, P. M., Defrère, D., et al. 2014, Proc. SPIE, 9147, 914710

Howard, A. W., \& Fulton, B. J. 2016, PASP, 128, 114401

Hunter, J. D. 2007, CSE, 9, 90

Ibukiyama, A., \& Arimoto, N. 2002, A\&A, 394, 927

Jones, J., White, R. J., Boyajian, T., et al. 2015, ApJ, 813, 58

Kelsall, T., Weiland, J. L., Franz, B. A., et al. 1998, ApJ, 508, 44

Kennedy, G. M., \& Piette, A. 2015, MNRAS, 449, 2304

Kennedy, G. M., \& Wyatt, M. C. 2013, MNRAS, 433, 2334

Kennedy, G. M., Wyatt, M. C., Bailey, V., et al. 2015, ApJS, 216, 23

Kharchenko, N. V., Scholz, R., Piskunov, A. E., Roeser, S., \& Schilbach, E. 2007, yCat, 3254, 0

Kirchschlager, F., Wolf, S., Krivov, A. V., Mutschke, H., \& Brunngräber, R. 2017, MNRAS, 467, 1614

Koerner, D. W., Kim, S., Trilling, D. E., et al. 2010, ApJL, 710, L26

Krist, J., Nemati, B., \& Mennesson, B. 2016, JATIS, 2, 011003

Krivov, A. V., Eiroa, C., Löhne, T., et al. 2013, ApJ, 772, 32

Lawler, S. M., Beichman, C. A., Bryden, G., et al. 2009, ApJ, 705, 89

Lebreton, J., Beichman, C., Bryden, G., et al. 2016, ApJ, 817, 165

Lebreton, J., van Lieshout, R., Augereau, J.-C., et al. 2013, A\&A, 555, A146

Lestrade, J.-F., \& Thilliez, E. 2015, A\&A, 576, A72

Lisse, C. M., Wyatt, M. C., Chen, C. H., et al. 2012, ApJ, 747, 93

Liu, W. M., Hinz, P. M., Hoffmann, W. F., et al. 2004, ApJL, 610, L125

MacGregor, M. A., Wilner, D. J., Andrews, S. M., Lestrade, J.-F., \& Maddison, S. 2015, ApJ, 809, 47

Mallik, S. V., Parthasarathy, M., \& Pati, A. K. 2003, A\&A, 409, 251

Mamajek, E. E., \& Hillenbrand, L. A. 2008, ApJ, 687, 1264

Marino, S., Wyatt, M. C., Panić, O., et al. 2017, MNRAS, 465, 2595

Marion, L., Absil, O., Ertel, S., et al. 2018, A\&A, submitted

Marshall, J. P., Krivov, A. V., del Burgo, C., et al. 2013, A\&A, 557, A58

Matthews, B. C., Sibthorpe, B., Kennedy, G., et al. 2010, A\&A, 518, L135+

Mennesson, B., Absil, O., Lebreton, J., et al. 2013, ApJ, 763, 119

Mennesson, B., Defrère, D., Nowak, M., et al. 2016a, Proc. SPIE, 9907, 99070X

Mennesson, B., Gaudi, S., Seager, S., et al. 2016b, Proc. SPIE, 9904, 99040L

Mennesson, B., Millan-Gabet, R., Serabyn, E., et al. 2014, ApJ, 797, 119

Mennesson, B., Serabyn, E., Hanot, C., et al. 2011, ApJ, 736, 14

Mérand, A., Bordé, P., \& Coudé du Foresto, V. 2005, A\&A, 433, 1155

Meshkat, T., Kenworthy, M. A., Reggiani, M., et al. 2015, MNRAS, 453, 2533

Millan-Gabet, R., Serabyn, E., Mennesson, B., et al. 2011, ApJ, 734, 67

Moerchen, M. M., Telesco, C. M., \& Packham, C. 2010, ApJ, 723, 1418

Montesinos, B., Eiroa, C., Krivov, A. V., et al. 2016, A\&A, 593, A51

Moran, S. M., Kuchner, M. J., \& Holman, M. J. 2004, ApJ, 612, 1163

Nesvorný, D., Jenniskens, P., Levison, H. F., et al. 2010, ApJ, 713, 816

Nuñez, P. D., Scott, N. J., Mennesson, B., et al. 2017, arXiv:1709.01655

Ochsenbein, F., Bauer, P., \& Marcout, J. 2000, A\&AS, 143, 23

Peterson, D. M., Hummel, C. A., Pauls, T. A., et al. 2006, Natur, 440, 896 Reidemeister, M., Krivov, A. V., Stark, C. C., et al. 2011, A\&A, 527, A57

Rieke, G. H., Gáspár, A., \& Ballering, N. P. 2016, ApJ, 816, 50

Rieke, G. H., Su, K. Y. L., Stansberry, J. A., et al. 2005, ApJ, 620, 1010

Roberge, A., Chen, C. H., Millan-Gabet, R., et al. 2012, PASP, 124, 799

Serabyn, E., Mennesson, B., Colavita, M. M., Koresko, C., \& Kuchner, M. J. 2012, ApJ, 748, 55

Smith, R., Wyatt, M. C., \& Haniff, C. A. 2009, A\&A, 503, 265 
Stark, C. C., \& Kuchner, M. J. 2008, ApJ, 686, 637

Stark, C. C., \& Kuchner, M. J. 2009, ApJ, 707, 543

Stark, C. C., Roberge, A., Mandell, A., et al. 2015, ApJ, 808, 149

Stark, C. C., Shaklan, S., Lisman, D., et al. 2016, JATIS, 2, 041204

Stock, N. D., Su, K. Y. L., Liu, W., et al. 2010, ApJ, 724, 1238

Su, K. Y. L., De Buizer, J. M., Rieke, G. H., et al. 2017, AJ, 153, 226

Su, K. Y. L., Rieke, G. H., Malhotra, R., et al. 2013, ApJ, 763, 118

Su, K. Y. L., Rieke, G. H., Stansberry, J. A., et al. 2006, ApJ, 653, 675

Thureau, N. D., Greaves, J. S., Matthews, B. C., et al. 2014, MNRAS, 445,2558

Trilling, D. E., Bryden, G., Beichman, C. A., et al. 2008, ApJ, 674, 1086

van Leeuwen, F. 2007, A\&A, 474, 653 van Lieshout, R., Dominik, C., Kama, M., \& Min, M. 2014, arXiv:1404.3271 Vican, L. 2012, AJ, 143, 135

Weinberger, A. J., Bryden, G., Kennedy, G. M., et al. 2015, ApJS, 216, 24

Wenger, M., Ochsenbein, F., Egret, D., et al. 2000, A\&AS, 143, 9

Wyatt, M. C. 2005, A\&A, 433, 1007

Wyatt, M. C., Greaves, J. S., Dent, W. R. F., \& Coulson, I. M. 2005, ApJ, 620,492

Wyatt, M. C., Smith, R., Greaves, J. S., et al. 2007, ApJ, 658, 569

Yoon, J., Peterson, D. M., Kurucz, R. L., \& Zagarello, R. J. 2010, ApJ, 708, 71

Yoon, J., Peterson, D. M., Zagarello, R. J., Armstrong, J. T., \& Pauls, T. 2008, ApJ, 681, 570

Zuckerman, B., Rhee, J. H., Song, I., \& Bessell, M. S. 2011, ApJ, 732, 61 